\title{
Electrolytic exfoliation of graphite in water with multifunctional electrolytes: en route towards high quality, oxide-free graphene flakes $\uparrow$
}

J.M. Munuera, J.I. Paredes, S. Villar-Rodil,* M. Ayán-Varela, A. Martínez-Alonso, J.M.D. Tascón

Instituto Nacional del Carbón, INCAR-CSIC, Apartado 73, 33080 Oviedo, Spain

* E-mail: silvia@incar.csic.es

$\dagger$ Electronic Supplementary Information (ESI) available: Experimental set-up for the electrolytic anodic treatments; visual and microscopic evidence for the expansion of graphite foil upon anodic treatment; evidence for the electrolytic treatment being the critical step in the exfoliation; additional AFM characterization of the samples; additional XPS characterization of the samples; measurement of film thickness by FE-SEM; derivatization and XPS analysis of the graphene flakes obtained by anodic treatments using SS as electrolyte; UHPLC/MS of the product of SNDS oxidation; demonstration for starting graphites other than graphite foil; growth of Pt NPs on electrolytically exfoliated graphene in the absence of amphiphile electrolyte/dispersants. See DOI:10.xxxx/cxxxxxxxx 


\begin{abstract}
Electrolytic - usually referred to as electrochemical - exfoliation of graphite in water under anodic potential holds enormous promise as a simple, green and high-yield method for the mass production of graphene, but currently suffers from several drawbacks that hinder its widespread adoption, one of the most critical being the oxidation and subsequent structural degradation of the carbon lattice that is usually associated to such a production process. To overcome this and other limitations, we introduce and implement the concept of multifunctional electrolytes. The latter are amphiphilic anions (mostly polyaromatic hydrocarbons appended with sulfonate groups) that play different relevant roles: (1) intercalating electrolyte to trigger exfoliation of graphite into graphene flakes, (2) dispersant to afford stable aqueous colloidal suspensions of the flakes suitable for further use, (3) sacrificial agent to prevent graphene oxidation during exfoliation and (4) linker to promote nanoparticle anchoring on the graphene flakes, yielding functional hybrids. The implementation of this strategy with some selected amphiphiles even furnishes anodically exfoliated graphenes of a quality similar to that of flakes produced by direct, ultrasound- or shear-induced exfoliation of graphite in the liquid phase (i.e., almost oxide- and defect-free). These high quality materials were used for the preparation of catalytically efficient graphene-Pt nanoparticle hybrids, as demonstrated by model reactions (reduction of nitroarenes). The multifunctional performance of these electrolytes is also discussed and rationalized, and a mechanistic picture of their oxidation-preventing ability is proposed. Overall, the present results open the prospect of anodic exfoliation as a competitive method for the production of very high quality graphene flakes.
\end{abstract}

\title{
1. Introduction
}

Boasting exceptional physical properties and a strong potential for application in many critical technological areas, including electronics, photonics, energy conversion/storage, catalysis and biomedicine, graphene is set to become one of the key materials of the next few decades. ${ }^{1-4}$ However, despite the enormous progress attained over the last years, issues related to the mass 
production of this two-dimensional form of carbon with on-demand specifications by costeffective means have not yet been settled and constitute a significant obstacle in the path of graphene-based disruptive technologies. ${ }^{4}$ In this endeavor, top-down fabrication approaches, which rely on the exfoliation of graphite and graphite derivatives, are the focus of considerable research efforts, as they offer the opportunity to access very large quantities of graphene flakes suitable for a wide range of prospective applications starting from an abundant and affordable source material. ${ }^{4-6}$

Currently, three main top-down methods of graphene production that are particularly promising in terms of scalability can be identified: ${ }^{7}$ (i) exfoliation and subsequent reduction of graphite oxide, (ii) direct exfoliation of graphite in the liquid phase, and (iii) electrolytic exfoliation of graphite. The graphite oxide route is particularly attractive in that it can provide very large quantities of single-layer sheets, in the form of both graphene oxide (GO) and reduced graphene oxide (RGO), which have been found useful in a range of potential applications. ${ }^{8-12}$ Nevertheless, the carbon lattice in GO and RGO is generally riddled with oxygen groups and structural defects derived from the harsh oxidation process, which have traditionally limited their wider utility in the graphene realm. ${ }^{7,13,14}$ On the other hand, very high quality graphene can be obtained by direct liquid-phase exfoliation of graphite, usually triggered by ultrasound waves or shear forces. ${ }^{15-17}$ This method naturally affords colloidal dispersions of defect-free graphene flakes that can be further processed into valuable materials, such as thin conductive films, polymer composites or hybrid structures, but on the downside it suffers from low exfoliation yields (typically $<1 \mathrm{wt} . \%$ ) and the as-prepared materials are frequently dominated by multilayer (mostly $3-8)$ rather than single- or few-layer $(<4)$ flakes. ${ }^{16,17}$ By contrast, electrolytic exfoliation of graphite has been shown to overcome, at least in part, some of the main limitations of the two previous approaches. ${ }^{18-20}$ Specifically, this method can provide graphene samples at relatively high yields consisting of single- and few-layer flakes of a structural quality better than that of reduced graphene oxide. As a result, electrolytically exfoliated graphenes have recently started to be explored towards their use as, e.g., electrodes for 
supercapacitors ${ }^{21,22}$ and lithium-sulfur batteries, ${ }^{23}$ metal-free catalysts, ${ }^{24}$ catalyst supports ${ }^{25}$ or electromagnetic interference shielding devices. ${ }^{26}$

Overall, electrolytic processes for the exfoliation of graphite into graphene can be of either cathodic or anodic nature. ${ }^{20}$ Cathodic exfoliation is mostly based on the use of lithium or alkylammonium salts dissolved in organic solvents (e.g., propylene carbonate or dimethyl sulfoxide) as the electrolytic medium, ${ }^{27-31}$ whereas anodic exfoliation is usually carried out in water in the presence of ionic liquids, acids or inorganic salts, ${ }^{24,25,32-36}$ being therefore more attractive from an environmental standpoint and also generally in terms of production rates. Nevertheless, the anodic approach currently suffers from some significant drawbacks. First, to be useful for further processing purposes the exfoliated materials frequently need to be dispersed in the liquid phase to afford colloidal suspensions. Due to their predominant hydrophobic nature, anodically exfoliated graphenes are typically dispersed in organic solvents such as $N, N-$ dimethylformamide, ${ }^{24,25,33-35}$ whose efficiency to colloidally stabilize graphene flakes has been established in earlier studies. ${ }^{37-39}$ For many prospective uses of this type of graphene, however, water would be the preferred dispersing medium, and in such a case the use of surfactants or other stabilizers becomes essential. ${ }^{15,16}$ Thus, the anodic preparation of graphene could be deployed to a greater advantage if chemical species with the ability to act efficiently both as an electrolyte during the anodic exfoliation and as a dispersant to stabilize the resulting graphene flakes in water were identified. Some previous work using, e.g., sodium dodecyl sulfate or copper phthalocyanine tetrasulfonic acid has suggested such a strategy to be feasible. ${ }^{40-42}$ However, systematic studies to identify effective species for this dual role, as well as to rationalize their performance and compare it by some quantifiable metric would be needed to make the most of this approach, but have not yet been undertaken.

Second, anodically exfoliated graphenes tend to be significantly oxidized due to the generation of reactive oxygen species from water at the graphite anode, their oxidation degree being generally comparable to that found for RGO. ${ }^{19,20}$ Similar to the case of the latter, this generally unwanted 
effect induces structural degradation in the resulting graphenes and compromises their application scope. To address this issue, researchers have explored some approaches to curtail the extent of graphene oxidation, for example the use of inorganic salts instead of acids as the electrolyte, ${ }^{34}$ certain additives in combination with the electrolyte ${ }^{43}$ or specific types of graphite as the anode. ${ }^{44}$ Nevertheless, when these improved graphenes are compared with their corresponding reference samples, such efforts are seen to be successful only to a limited degree. Indeed, no anodic exfoliation strategy reported to date has afforded graphene flakes that exhibit a quality similar to that of flakes obtained by direct liquid-phase exfoliation of graphite, i.e. graphenes that are essentially oxide- and defect-free. Attaining such a goal would thus constitute a significant step forward.

With the aim of providing a unified solution to the abovementioned issues, we have conducted an investigation into a pool of electrolytes towards the anodic exfoliation of graphite into graphene, the results of which are reported here. First, a number of readily available amphiphilic anions are demonstrated to be effective as both exfoliating electrolyte and dispersant, easily affording aqueous colloidal suspensions of well-exfoliated graphene flakes. The performance of these chemical species in such a dual role is quantitatively compared and rationalized. Second, several of the newly identified electrolytes are also shown to prevent oxidation of the graphene flakes to a large extent, an ability that can be related to their propensity to get themselves oxidized by reactive oxygen species. Moreover, graphene flakes with chemical make-up and structural quality comparable to those typical of samples produced by direct ultrasonic or shear exfoliation of graphite are obtained using the best electrolytes. Finally, adding to the multifunctional nature of these electrolytes, we demonstrate the growth of Pt nanoparticles (NPs) onto the electrolyte-stabilized graphene flakes. The resulting graphene-Pt NP hybrids were seen to possess a remarkably high catalytic activity, as evaluated on the basis of model reduction reactions with nitroarenes.

\section{Experimental}




\subsection{Materials and reagents}

High purity graphite foil (Papyex I980, acquired from Mersen) was used as starting material throughout the study. The following chemical species were tested as electrolyte in the anodic exfoliation of graphite in aqueous medium: sodium sulfate (SS), sodium dodecyl sulfate (SDS), sodium methanesulfonate (SMS), sodium benzenesulfonate (SBS), disodium benzene-1,3disulfonate (SBDS), sodium $p$-toluenesulfonate (STS), sodium $p$-toluate (SPT), sodium dodecylbenzene sulfonate (SDBS), disodium naphthalene-1,5-disulfonate (SNDS), disodium naphthalene-2,6-dicarboxylate (SNDC), trisodium naphthalene-1,3,(6,7) -trisulfonate (SNTS), sodium pyrene-1-sulfonate (SPS) and tetrasodium pyrene-1,3,6,8-tetrasulfonate (SPTS). These chemicals were obtained from Sigma-Aldrich, either as such or in their corresponding acid form, which was then converted to the sodium salt by mixing with the appropriate molar amount of $\mathrm{NaOH}$.

\subsection{Anodic exfoliation and dispersion of graphite in water with different electrolytes}

The anodic exfoliation process was carried out in a two-electrode configuration, using a graphite foil piece $\left(40 \times 25 \times 0.5 \mathrm{~mm}^{3}\right)$ as working electrode and a platinum wire as counter electrode. The two electrodes were submerged in an aqueous solution $(20 \mathrm{~mL})$ of a given electrolyte at a certain concentration (specified below), and then a positive voltage $(10 \mathrm{~V})$ was applied to the graphite electrode for 60 min using an Agilent 6614C DC power supply. The platinum wire was placed parallel to the graphite foil surface at a distance of about $2 \mathrm{~cm}$. A digital photograph of the experimental set-up is depicted in Fig. S1 of the ESI $\uparrow$. During the electrolytic treatment, the graphite sample was typically seen to swell and expand. Visual and microscopic evidence for such expansion are given in Fig. S2 of the ESI $\dagger$. After the 60 min electrolysis period, the resulting expanded product was gently scraped with a spatula from its parent graphite piece and transferred directly to the working aqueous solution. The latter was subsequently sonicated for $3 \mathrm{~h}$ in an ultrasound bath cleaner (J.P. Selecta Ultrasons system, $40 \mathrm{kHz}$ ). The obtained dispersion was purified by two cycles of sedimentation via centrifugation $(20000 g, 20 \mathrm{~min}$; Eppendorf 5424 
microcentrifuge) and re-suspension in Milli-Q water with a brief (2-3 min) sonication, and finally subjected to a mild centrifugation step $(200 \mathrm{~g}, 20 \mathrm{~min})$ to remove the non-exfoliated or poorly exfoliated graphite fraction from the resulting supernatant, which was collected for further use. The concentration of graphene in the final supernatant was taken as a metric for the overall efficiency of the investigated chemical species in the dual role of exfoliating (intercalating) electrolyte and dispersant. Following previously reported protocols, the concentration was determined by UV-vis absorption spectroscopy (double-beam He $\lambda$ ios $\alpha$ instrument, from Thermo Spectronic) based on the Lambert-Beer law and using a value of $2440 \mathrm{~mL} \mathrm{mg}^{-1} \mathrm{~m}^{-1}$ for the extinction coefficient at the measured wavelength of $660 \mathrm{~nm} .^{45,46}$

\subsection{Synthesis and catalytic activity of anodically exfoliated graphene-Pt NP hybrids}

Graphene-Pt NP hybrids were prepared from several of the previously obtained aqueous graphene dispersions by the following procedure: $9 \mathrm{~mL}$ of a given anodically exfoliated graphene suspension $\left(0.2 \mathrm{mg} \mathrm{mL}^{-1}\right)$ were heated at $85^{\circ} \mathrm{C}$ under magnetic stirring in a $20 \mathrm{~mL}$ test tube sealed with a septum. Then, $1 \mathrm{~mL}$ of $1.1 \mathrm{mM}$ aqueous $\mathrm{K}_{2} \mathrm{PtCl}_{4}$ solution was added and the mixture was magnetically stirred at the mentioned temperature for $2 \mathrm{~h}$. To remove unreacted Pt salt, the resulting product was centrifuged at $20000 \mathrm{~g}$ for $20 \mathrm{~min}$ and resuspended in Milli-Q water via sonication for 1-2 min. Subsequently, $20 \mu \mathrm{L}$ of $1.5 \mathrm{M} \mathrm{NaBH}_{4}$ were added to the suspension and allowed to react for $45 \mathrm{~min}$ at room temperature. Following this step, the final product was purified by two cycles of sedimentation via centrifugation (20000 $\mathrm{g}, 20 \mathrm{~min})$ and resuspension in Milli-Q water. The catalytic activity of the obtained graphene-Pt NP hybrids was evaluated on the basis of two model reactions with nitroarenes: the reduction of 4-nitrophenol (4-NP) and 4-nitroaniline (4-NA) to 4aminophenol (4-AP) and $p$-phenylenediamine ( $p$-PDA), respectively, in aqueous medium at room temperature using $\mathrm{NaBH}_{4}$ as a reductant. To this end, mixed aqueous solutions containing $0.06 \mathrm{mM}$ of the nitroarene, $36 \mathrm{mM}$ of $\mathrm{NaBH}_{4}$ and $1.8-4.8 \mu \mathrm{g} \mathrm{mL}^{-1}$ of graphene flakes decorated with Pt NPs (amounting to concentrations of the latter of $0.8-2.1 \mu \mathrm{M}$ ) were freshly prepared and the reaction 
progress was monitored by measuring their optical density at a given wavelength at $0.25 \mathrm{~s}$ intervals with UV-vis absorption spectroscopy. For reasons that will be discussed below, the monitored wavelengths were 400 and $380 \mathrm{~nm}$ in the case of 4-NP and 4-NA, respectively.

\subsection{Characterization techniques}

Characterization of the samples was accomplished by UV-vis absorption spectroscopy, Raman spectroscopy, X-ray photoelectron spectroscopy (XPS), scanning transmission electron microscopy (STEM), transmission electron microscopy (TEM), atomic force microscopy (AFM), inductively coupled plasma-mass spectrometry (ICP-MS) and measurement of electrical conductivity. Raman spectra were recorded with a Horiba Jobin-Yvon LabRam instrument using a laser excitation wavelength of $532 \mathrm{~nm}$ at an incident power of $2.5 \mathrm{~mW}$. XPS measurements were performed on a SPECS apparatus under a pressure of $10^{-7} \mathrm{~Pa}$ with a non-monochromatic $\mathrm{Mg} \mathrm{K}_{\alpha} \mathrm{X}$-ray source $(11.81 \mathrm{kV}, 150 \mathrm{~W})$. Samples for both Raman spectroscopy and XPS were prepared by drop-casting the corresponding suspension onto a pre-heated $\left(\sim 50-60^{\circ} \mathrm{C}\right)$ circular stainless steel sample-holder $12 \mathrm{~mm}$ in diameter and allowing it to dry. STEM imaging was accomplished with a Quanta FEG 650 system (FEI Company) operated at $30 \mathrm{kV}$, while TEM images were acquired on a JEOL 2000 EX-II instrument operated at $160 \mathrm{kV}$. For STEM and TEM, specimens were prepared by mixing the corresponding suspension with an equal volume of ethanol, and then $40 \mu \mathrm{L}$ of the resulting dispersion were drop-cast onto a copper grid (200 square mesh) covered with a carbon film (Electron Microscopy Sciences) and allowed to dry under ambient conditions. AFM imaging was conducted with a Nanoscope IIIa Multimode apparatus (Veeco Instruments) under ambient conditions in the tapping mode of operation. To this end, rectangular silicon cantilevers with nominal spring constant and resonance frequency values of $40 \mathrm{~N} \mathrm{~m}^{-1}$ and $250-300 \mathrm{kHz}$, respectively, were employed. Either highly oriented pyrolytic graphite (HOPG) pieces or $\mathrm{SiO}_{2}(300$ $\mathrm{nm}) / \mathrm{Si}$ wafers were used as substrates for AFM. The substrates were pre-heated at $\sim 50-60{ }^{\circ} \mathrm{C}$ and then a small volume $(20-40 \mu \mathrm{L})$ of graphene dispersion $\left(\sim 0.05 \mathrm{mg} \mathrm{mL}^{-1}\right)$ was drop-cast and 
allowed to dry. ICP-MS analysis of graphene-Pt NP hybrids was carried out with a 7500ce apparatus (Agilent) furnished with an octopole collision/reaction cell to destroy interfering ions. Before analysis, the samples were subjected to microwave-assisted acid digestion. To measure electrical conductivity, the graphene samples were processed into free-standing paper-like films by vacuum filtration of the corresponding colloidal dispersions through silver membrane filters $25 \mathrm{~mm}$ in diameter and $0.2 \mu \mathrm{m}$ of pore size (Sterlitech Corporation). Then, $12 \times 12 \mathrm{~mm}^{2}$ square pieces were cut from the films and their conductivity was determined through the van der Pauw method by means of a homemade setup (Agilent $6614 \mathrm{C}$ potentiostat and Fluke 45 digital multimeter). The thickness of the films was estimated by field-emission scanning electron microscopy (FE-SEM, Quanta FEG 650 system, from FEI Company).

\section{Results and discussion}

\subsection{Electrolytes with dual functionality towards the anodic exfoliation and dispersion of graphite in} water

As a first step in the investigation, we sought to identify chemical species that function both as efficient electrolytes to promote the anodic exfoliation of graphite in water and as dispersants to colloidally stabilize the resulting graphene flakes. Therefore, some rationale had to be first established in the selection of the potential candidates that were subsequently evaluated. To this end

we note that, according to previous reports, ${ }^{32,34}$ the anodic exfoliation of graphite in aqueous electrolytic solution relies on the following two basic processes: (1) pre-expansion of the graphite layers, starting at edge sites, through attack by hydroxyl and oxygen radicals generated from the oxidation of water molecules at the anode; (2) subsequent separation of the pre-expanded layers (i.e., exfoliation) as a result of the intercalation of anions from the electrolyte. This mechanism implies that successful chemical species that can both exfoliate and colloidally stabilize graphite by the approach investigated here have to be of anionic nature. In addition, it is well known that the essentially hydrophobic character of graphene requires the use of amphiphilic molecules for it to be 
colloidally stabilized in water, and anodically exfoliated graphene is no exception in this regard. ${ }^{15,16}$ Such amphiphilic dispersants typically consist of a non-polar, hydrophobic component that adsorbs onto the graphene surface and a polar, hydrophilic moiety that extends into the aqueous medium. ${ }^{16,47,48}$ With these considerations in mind, we selected a number of molecules incorporating a hydrophobic (aromatic- and/or alkyl-containing) section appended with one or several anionic groups that were intended to serve both as the polar moiety required to make up the amphiphilic structure and as the anions that drive the intercalation of the molecule during the electrolytic process. Although several anions have been previously tested for electrolytic intercalation of graphite (e.g., sulfate, phosphate, nitrate or tetrafluoroborate), ${ }^{24,32-34,49}$ sulfates and sulfonates were mostly chosen for the present work, as they are known to be particularly efficient towards this purpose and can also be readily combined with organic moieties to form amphiphilic molecules.

Table 1 lists the name, chemical structure and acronym used for the species tested here as potential electrolyte/dispersant, which generally comprise an alkyl (e.g., dodecyl) or aromatic (benzene, naphthalene or pyrene) moiety appended with one, two, three or four sulfonate groups. We note that some of these molecules have already been used as dispersants in the direct exfoliation (via sonication) of graphite to give graphene. ${ }^{47,50,51}$ However, the high efficacy of a given amphiphile as a graphene dispersant is not expected to guarantee its success as intercalating electrolyte and, therefore, that it can be considered an efficient electrolyte/dispersant overall. To provide some quantitative measure of overall performance, we carried out benchmark tests based on a protocol that comprised the following steps (described in detail in the Experimental section): (1) anodic treatment of a graphite piece of set dimensions in aqueous solution of a given chemical species; (2) bath sonication of the anodically expanded product in the same solution; (3) purification of the resulting dispersion by sedimentation via centrifugation and subsequent re-suspension in Milli-Q water; (4) determination of the concentration of graphene material in the final dispersion by UV-vis absorption spectroscopy. The results of such tests performed for the different chemical species at various molar concentrations are summarized in Table 1. Before discussing them in detail, 
we note that in many cases the described preparation protocol led to homogeneous, opaque black suspensions, as exemplified in Fig. 1a with SNTS, SPTS and SNDS, which were seen to be colloidally stable for weeks to months. Fig. 1b shows some representative UV-vis absorption spectra of the dispersions. The spectra were dominated by an intense band located at $\sim 270 \mathrm{~nm}$, together with strong absorbance in the 300-1000 nm wavelength range. These features were nearly identical to those observed previously for both reduced graphene oxide and pristine graphene flakes, ${ }^{47,52,53}$ and are known to reveal the presence of graphitic material possessing extended electronically conjugated domains. Additional bands of varied intensity located in the 200-400 nm wavelength range were also quite frequently noticed in the spectra of the obtained suspensions. Control experiments indicated that they were associated to the chemical species used as electrolyte/dispersant, and more specifically to their aromatic moieties $\left(\pi \rightarrow \pi^{*}\right.$ transitions $) .{ }^{54}$

Focusing now on the results of the tests (Table 1), we first note that a number of the assayed chemical species were not successful in yielding aqueous dispersions with non-negligible content of graphitic material (i.e., entries in Table 1 that indicate zero graphene concentration), even though some of these species were known to be very efficient either as electrolyte in the anodic exfoliation of graphite or as graphene dispersant. For example, SS afforded a large amount of expanded product upon anodic treatment, in agreement with previous results from the literature, ${ }^{21,34}$ but the sulfate anion alone failed to colloidally stabilize the resulting graphene sheets due to the lack of a hydrophobic moiety that could adsorb onto the sheets. Incorporation of a methyl unit to the anion did not suffice to provide the electrolyte with the sought-after quality of colloidal stabilizer, as demonstrated by the fact that SMS, while inducing also a vigorous anodic expansion of the graphite piece (see Fig. S2 in ESI†), was not able to afford final aqueous dispersions possessing any significant amount of graphitic product. We interpret that, having such a short alkyl chain, SMS is only very weakly adsorbed on the graphene sheets and thus cannot confer them enough colloidal stability. Likewise, SPS, which is made up of a sulfonate group appended to a pyrene unit, has been shown to be a powerful dispersant of graphene flakes in aqueous medium, ${ }^{50,51}$ but in the present 
work could not be put to good use as electrolyte for the anodic expansion of graphite. The graphite foil sample did not expand to any significant extent in aqueous solutions of this species, and its surface was just seen to roughen after the $60 \mathrm{~min}$ electrolytic treatment. We note that, due to solubility limitations, SPS could be tested only at concentrations of up to $0.01 \mathrm{M}$, whereas sulfate salts (e.g., SS and others) in successful anodic exfoliations have been typically used at concentrations in the range of $0.1 \mathrm{M}^{33-35} \mathrm{We}$ conclude that just a very limited degree of intercalation is possible with SPS at the attainable concentrations, being clearly insufficient to induce a significant expansion, and then exfoliation, of the graphite anode.

The previous results suggest that chemical species must possess appropriate combinations of certain characteristics to be successful in the dual role of electrolyte and dispersant. For instance, their hydrophobic moiety should be large enough to allow sufficiently strong adsorption on the graphene flakes, but not too bulky so as to hamper the electrophoretic mobility of the amphiphilic anion during the electrolytic process (as determined by its charge/mass ratio) and/or its ability to intercalate between the graphene layers. In this regard, a single benzene unit coupled to the sulfonate anion was already seen to furnish the resulting amphiphilic molecule (i.e., SBS) with a non-negligible stabilizing capability, presumably due to the establishment of a relatively strong interaction $(\pi-\pi)$, while retaining at the same time the ability of SS and SMS to expand graphite upon electrolytic treatment. As a result, SBS yielded stable aqueous suspensions of graphitic material at significant concentrations. A similar result was obtained with a sulfonate group attached to a toluene moiety (STS). Likewise, using amphiphiles that incorporate several, rather than one, ionic groups could be an effective strategy to settle the aforementioned issues, at least in part. Support for such a hypothesis was obtained by noting that, contrary to the case of SPS, SPTS (i.e., a pyrene molecule with four sulfonate groups attached; see Table 1) afforded large amounts of anodically expanded material, even if working at SPTS concentrations of $0.01 \mathrm{M}$ and below. The expanded product could then be colloidally stabilized at reasonable concentrations. It is worth mentioning that SPTS is substantially less efficient than SPS as a graphene dispersant, ${ }^{50,51}$ but this 
relative drawback was more than outweighed by the fact that it was quite effective in inducing the anodic expansion of graphite, whereas SPS was not.

Similarly, SBDS (a benzene ring coupled to two sulfonate groups) tended to outperform SBS as electrolyte/dispersant overall (higher concentration of graphitic material in the final dispersion) at electrolyte concentrations of 0.01 and $0.05 \mathrm{M}$; however, the use of higher electrolyte concentrations (i.e., 0.1 and $0.2 \mathrm{M}$ ) led to similar or even better performance for SBS. To shed an understanding on this question, we carried out a control experiment to determine which of the two amphiphiles performs better as a graphene dispersant. To this end, we first prepared a certain amount of anodically expanded graphite using SS $(0.1 \mathrm{M})$ as the electrolyte. After extensive washing and drying, equal amounts of the obtained product were dispersed in aqueous solutions of SBS and SBDS at different concentrations between 0.01 and $0.2 \mathrm{M}$, bath-sonicated for $3 \mathrm{~h}$ and finally centrifuged. It was apparent to the naked eye, but also confirmed by UV-vis absorption spectroscopy, that the amount of graphitic material retained in the resulting supernatants was substantially larger when using SBS for all the tested concentrations, i.e. this amphiphile was far superior to SBDS as a dispersant. Such a result parallels the observations on the performance of SPS and SPTS as graphene dispersants reported and rationalized by other authors, as mentioned above, and can be explained by invoking a similar line of reasoning: ${ }^{51}$ the ability of these amphiphilic anions to colloidally stabilize graphene sheets in aqueous medium is expected to be governed by the strength of their adsorption onto the sheets, i.e., by their adsorption free energy. The latter is in turn determined by the interplay between the solvation free energy of the amphiphile in water and the interaction free energy of the amphiphile with the graphene surface. The interaction free energy of SBS and SBDS with graphene can be assumed to be similar or even somewhat larger for SBDS on account of its additional sulfonate group. However, this additional group would make the solvation free energy of SBDS to be substantially larger and hence its adsorption free energy to be smaller than their counterparts for SBS. As a result, SBDS would exhibit a weaker ability to colloidally stabilize graphene in water, as was indeed observed in the control experiment. 
In the light of the previous discussion, the following rationale can be put forward to account for the observed behavior of SBS and SBDS towards their combined role of electrolyte/dispersant. At low electrolyte concentration, the expected higher electrophoretic mobility of SBDS will lead to a larger extent of intercalation of the graphite anode, and consequently to a much larger amount of expanded product (as was visually evident), compared with the case of SBS. As a result, SBDS will afford more graphitic material in the final dispersion simply because there was very little expanded product available for dispersion with SBS (see Table 1). On the other hand, it can be argued that the low intercalation rate of the SBS molecule arising from its comparatively low mobility can be compensated for when working at high electrolyte concentrations. In such a case, the sheer amount of SBS used would be enough to induce a sizable intercalation and expansion of the graphite anode, as was also observed in the experiments. With a significant amount of expanded graphite in the aqueous medium, the concentration of graphitic material in the final suspension after the sonication step will most probably be dictated by the ability of the amphiphile to act as a dispersant, so in this case higher concentrations would be expected when using SBS compared to SBDS, as it was indeed observed.

A limited electrophoretic mobility of amphiphiles that incorporate a single anion and a relatively bulky hydrophobic moiety, therefore possessing relatively low charge/mass ratios, can also be invoked to explain why SDS (sulfate group with a dodecyl chain) and SDBS (sulfonate group with a dodecylbenzene moiety), which are known to be reasonably efficient surfactants for graphene, ${ }^{47,55}$ did not succeed in expanding the graphite anode to any significant extent at low electrolyte concentration $(0.01 \mathrm{M})$, similar to what was observed for SPS. However, contrary to the case of the latter species and due to their ability to assemble into micelles, both SDS and SDBS could be tested as electrolytes at concentrations well above $0.01 \mathrm{M}$. In line with the ideas discussed in the preceding paragraph for SBS, the use of sufficiently high concentrations of these species (e.g., 0.05 M for both SDS and SDBS) led to a substantial expansion of the graphite anode and finally to aqueous dispersions containing considerable amounts of graphitic material (see Table 1). We note 
that all the concentrations tested for SDS and SDBS (see Table 1) were above their corresponding critical micelle concentration values, ${ }^{56}$ which warranted the effectiveness of both surfactants in the colloidal stabilization of the expanded graphitic products.

The information obtained from the experimental results presented above can be used as a guide to select further amphiphiles that are effective in the sought-after dual role of electrolyte and dispersant. Such results suggest that successful candidates must strike an appropriate balance between their net charge and the mass of their hydrophobic moiety. On one hand, the net charge should be high enough to promote efficient intercalation/ expansion of the graphite anode but not too high so as to critically weaken the free energy of adsorption of the amphiphile on the graphene surface. On the other hand, the hydrophobic component should be large enough to favour its adsorption onto the graphene surface, but not too large so as to hamper the electrophoretic mobility of the molecule. More specifically, if SPS suffers from solubility and electrophoretic mobility limitations, but both SPTS and SBS possess appropriate combinations of the desired characteristics, then it is reasonable to expect that amphiphilic anions comprising two or three benzene rings as the hydrophobic moiety and two or three sulfonate groups should also perform efficiently as electrolyte and dispersant. To corroborate this point, we tested two chemical species that consisted of a naphthalene unit appended with either two or three sulfonate groups (i.e., SNDS and SNTS, respectively), and in both cases stable aqueous suspensions containing significant amounts of graphitic material could be readily obtained even at low electrolyte concentrations (see Table 1). As a final comment on the competence of the tested species to act as efficient electrolytes, it is worth mentioning that the nature of the anionic group present in the amphiphile was central to its ability to intercalate the graphite electrode. We selected sulfonate- and sulfate-containing amphiphiles based on the known propensity of graphite to be intercalated by such anions, both electrolytically and by other means. ${ }^{57-59}$ Indeed, as exemplified with the case of SPT (toluene with a carboxylate) and SNDC (naphthalene with two carboxylates), replacement of the sulfonate groups by carboxylates led to chemical species that could not efficiently intercalate and expand the graphite anode, even 
under basic conditions to ensure that they were in a fully deprotonated, negatively charged state. This result is in agreement with previous results indicating that the use of, e.g., acetic or formic acid as electrolyte did not result in any significant exfoliation of the graphite anode. ${ }^{24}$ Finally, the exfoliation yield for the electrolytic process investigated here with the best performing electrolytes was $\sim 10 \mathrm{wt}$. \% which significantly exceeded that obtained by liquid phase exfoliation of graphite via ultrasonication in sodium cholate (SC) aqueous solutions for similar treatment times of 4 hours $(\sim 0.5$ wt. $\%){ }^{60}$

\subsection{Chemical and structural characterization of the exfoliated products: evidence for high quality} graphene flakes with very low oxygen content

To elucidate the morphological (exfoliation degree, object shape/size), chemical (e.g., oxidation level) and structural characteristics of the exfoliated materials using the electrolytes/dispersants discussed above, STEM, AFM, XPS and Raman spectroscopy measurements were carried out. Fig. $2 \mathrm{a}$ and $\mathrm{b}$ shows STEM images of the dispersions obtained with $0.2 \mathrm{M}$ SNDS (a) and 0.01 M SPTS (b), where the effective exfoliation of the starting graphite into thin flakes was made evident. This was further supported by AFM imaging, as illustrated in Fig. 2c-f for selected dispersions after being drop-cast onto HOPG substrates, more to the point those prepared with $0.1 \mathrm{M} \mathrm{SS}$ (c, dispersion prepared in water-isopropanol mixture and shown for comparison purposes), $0.01 \mathrm{M}$ SPTS (d), 0.05 M SBDS (e) and 0.2 M SNDS (f). For all the successful electrolytes/dispersants, the resulting suspensions were comprised of very thin and flat objects displaying irregular polygonal shapes, consistent with them being well exfoliated graphene flakes. We note that in Fig. 2 d-f a high density of small rounded (as opposed to polygonal) features decorate the HOPG surface. These can be attributed to the amphiphilic dispersants, which are expected to strongly adsorb onto the HOPG surface as well as onto the graphene flakes. The flakes exhibited typical lateral dimensions between a few and several hundred nanometers, and their apparent thickness, determined as flake height relative to the substrate (see exemplary line profiles in Fig. $2 \mathrm{~d}-\mathrm{f}$ ), ranged between $\sim 0.5$ and $2.5 \mathrm{~nm}$, although the specific values differed slightly depending on the particular electrolyte/dispersant used 
(see Table 1 and Fig. S4 of the ESI $\dagger$ ). These values were very similar to the ones reported here and in the literature for graphene flakes anodically exfoliated in sulphuric acid or sulfate salts (e.g., SS), ${ }^{33,44,61,62}$ suggesting that intercalation of the graphite electrode was not critically hampered by the larger size of the (amphiphilic) anions used here. We also note that the apparent thickness values were well below those usually reported for surfactant-stabilized graphene dispersions obtained by direct exfoliation of graphite via sonication or shear mixing $(\sim 3-8 \mathrm{~nm}),{ }^{17,46}$ thus suggesting a much better degree of exfoliation. In fact, taking into account that the typical thickness of the patches of amphiphilic molecules adsorbed onto the HOPG surface (Fig. 2d-f) was slightly above $1 \mathrm{~nm}$ and that these molecules are expected to contribute to the measured thickness of the flakes, we conclude that all the samples obtained here are mostly made up of single- to few-layer $(<5)$ flakes.

The chemical make-up of the exfoliated graphene flakes was investigated by XPS. The recorded survey spectra (not shown) indicated that carbon was the dominant element for all the samples, as expected, but oxygen and sulphur were also present to a variable extent depending on the specific amphiphile used (see ESI† for further details on XPS analysis). We note that the graphene dispersions employed for the XPS measurements were subjected to a special purification protocol consisting of several cycles of sedimentation via centrifugation and re-suspension in Milli-Q water. This procedure aimed at removing the largest possible amount of amphiphile from the samples, so that the actual oxygen content of the graphene flakes could be properly determined without substantial interference from the sulfonate/sulfate groups of the electrolyte/dispersant. Table 1 lists the oxygen content, expressed as $\mathrm{O} / \mathrm{C}$ atomic ratio, for the different graphene samples prepared with the successful amphiphiles as well as with SS $(0.1 \mathrm{M})$, the latter serving as a benchmark to afford direct comparisons with an electrolyte commonly used in the anodic exfoliation of graphite. As could be anticipated, the SS-derived graphene flakes were seen to be significantly oxidized $(\mathrm{O} / \mathrm{C}$ ratio $\sim 0.11)$. Similar or even higher levels of oxidation are usually reported for anodically exfoliated graphene, ${ }^{21,24,25,49,62,63}$ which are thought to be the result of 
oxidation reactions inherent to such an exfoliation process carried out in aqueous medium. More specifically, it has been postulated that water molecules are readily oxidized at the graphite anode, giving rise to highly reactive hydroxyl and oxygen radicals that then attack the graphene layers. ${ }^{32,34}$ Thus, a more or less extensive oxidation of the resulting graphenes has been generally regarded as an inescapable consequence of the anodic exfoliation approach. ${ }^{19,20}$ Because oxidation is almost invariably associated to degradation of the structural quality and properties of graphene, anodically exfoliated graphenes are deemed to have a relatively narrow scope of application compared to that of high quality graphene flakes obtained, e.g., by cathodic exfoliation of graphite in organic solvents or direct exfoliation in the liquid phase via ultrasound or shear forces.

Nevertheless, the present results indicated that graphenes with limited oxidation could be attained with a proper choice of the electrolyte/dispersant. Although some amphiphiles (e.g., SBDS, SBS or SDBS) yielded products with a similar or somewhat lower O/C ratio than that of the SSderived material (see Table 1), others (e.g., SPTS, SNTS and SNDS) led to graphene samples with significantly reduced levels of oxidation. SNDS was particularly effective in this regard, affording $\mathrm{O} / \mathrm{C}$ ratios as low as 0.02 . To the best of our knowledge, this is by far the lowest degree of oxidation reported to date for any anodically exfoliated graphene, and is even lower than that typical of graphenes obtained by cathodic exfoliation approaches in organic solvents $(\mathrm{O} / \mathrm{C}$ ratios $\sim 0.04-$ 0.09). ${ }^{28-31,64}$ As illustrated in Fig. 3, the extent of oxidation of the graphene lattice was reflected in the shape of the high resolution $\mathrm{C}$ 1s core level XPS spectrum. For samples with high O/C ratios, e.g. those obtained with SS (a) and SBDS (b), the C 1s spectrum was dominated by a component centered at $\sim 284.6 \mathrm{eV}$, which can be attributed to carbon atoms in non-oxidized graphitic environments $(\mathrm{C}=\mathrm{C}$ species$)$, with an additional significant component located at about $286.5 \mathrm{eV}$ (C-O species in, e.g., hydroxyl or epoxide groups) and a minor one at $\sim 288.5 \mathrm{eV}$ (C=O species; e.g., carbonyls). ${ }^{44,62}$ We note that the combined weight of the two latter components was commensurate with the $\mathrm{O} / \mathrm{C}$ atomic ratio of the corresponding samples as deduced from their survey spectra. On the other hand, the weight of the $\mathrm{C}-\mathrm{O}$ and $\mathrm{C}=\mathrm{O}$ components (relative to the $\mathrm{C}=\mathrm{C}$ component) was 
seen to decrease substantially for graphene samples with reduced extent of oxidation (e.g., Fig. 3c for SPTS with O/C ratio of 0.06), and was virtually negligible for the SNDS-derived graphene with $\mathrm{O} / \mathrm{C}$ ratio $\sim 0.02$ (Fig. 3d, black trace). For comparison purposes, the $\mathrm{C} 1 \mathrm{~s}$ envelope of a graphene sample prepared by direct exfoliation of graphite powder through sonication in a sodium cholate aqueous solution, which is known to yield essentially pristine, oxide-free flakes, ${ }^{65}$ is also shown in Fig. 3d (red trace), being almost identical to that of the SNDS-derived graphene. O/C ratios of $\sim 0.01$ were measured for this pristine graphene sample, as well as for both its parent graphite powder and the graphite foil used for anodic exfoliation. These results indicate that oxidation of anodically exfoliated graphene can be largely prevented as long as a suitable electrolyte is used (e.g., SNDS). This is in sharp contrast with what has been usually found for this production method using common electrolytes (e.g., $\mathrm{H}_{2} \mathrm{SO}_{4}, \mathrm{~K}_{2} \mathrm{SO}_{4}$ or SS).

Further evidence of the high structural quality that could be attained with some of the tested amphiphiles was gathered by Raman spectroscopy. The same aforementioned purification procedure applied to samples for XPS analysis was used here. Some exemplary spectra are presented in Fig. 4 for graphenes anodically exfoliated with SS (a), SBDS (b), SPTS (c) and SNDS (d), as well as the graphene sample prepared by direct exfoliation of graphite in water-isopropanol mixture (e), shown for comparison. The first-order region of the spectra $\left(1100-1700 \mathrm{~cm}^{-1}\right)$ was dominated by the well-known G and D bands of graphitic materials, located at about 1582 and $1350 \mathrm{~cm}^{-1}$, respectively, while the so-called $2 \mathrm{D}$ band $\left(\sim 2700 \mathrm{~cm}^{-1}\right)$ was the main feature observed in the second-order region $\left(2300-3300 \mathrm{~cm}^{-1}\right)$. As noticed from Fig. 4, the intensity of the D band relative to the $\mathrm{G}$ band was seen to differ significantly between the different samples. Because the D peak is a defect-related band, whereas the $\mathrm{G}$ peak is just associated to the presence of $\mathrm{sp}^{2}$ carbon atoms, the integrated intensity ratio of the $\mathrm{D}$ and $\mathrm{G}$ bands $\left(\mathrm{I}_{\mathrm{D}} / \mathrm{I}_{\mathrm{G}}\right.$ ratio) is frequently taken as a quantitative measure of the structural disorder in graphite/graphene. ${ }^{66,67}$ The $\mathrm{I}_{\mathrm{D}} / \mathrm{I}_{\mathrm{G}}$ ratio was calculated for the different graphene samples and the results are given in Table 1. As could be expected, graphenes with high $\mathrm{O} / \mathrm{C}$ ratios tended to exhibit rather high values of $\mathrm{I}_{\mathrm{D}} / \mathrm{I}_{\mathrm{G}}$, indicative of 
carbon lattices with substantial structural disorder, whereas those samples with lower degrees of oxidation possessed correspondingly low $\mathrm{I}_{\mathrm{D}} / \mathrm{I}_{\mathrm{G}}$ ratios. In particular, the graphene material with the lowest O/C ratio (0.02), prepared using $\operatorname{SNDS}(0.2 \mathrm{M})$, exhibited an $\mathrm{I}_{\mathrm{D}} / \mathrm{I}_{\mathrm{G}}$ value of 0.2 , i.e., it was much smaller than that obtained with a standard electrolyte (SS, 1.0) but very similar to that of the graphene sample prepared by direct exfoliation of graphite $(\sim 0.2)$. This is clear indication that extensive introduction of disorder in the graphene lattice is not invariably associated to the anodic exfoliation process in aqueous medium, but graphenes with a very low defect content are also possible under certain preparation conditions (e.g., using appropriate electrolytes in the present case). As a result, materials with attractive characteristics (e.g., a high electrical conductivity) should be attainable using the high quality anodically exfoliated graphenes. For instance, the electrical conductivity of free-standing, paper-like graphene films prepared by vacuum filtration of dispersions derived from SNDS $(0.2 \mathrm{M})$ and SPTS $(0.01 \mathrm{M})$ was 46000 and $45000 \mathrm{~S} \mathrm{~m}^{-1}$ (see ESI $\dagger$ for details on film thickness by FE-SEM), which compares favorably with values previously reported for graphene films obtained by direct exfoliation of graphite via ultrasonication or shear mixing in organic solvents or water-surfactant systems $\left(2000-20000 \mathrm{~S} \mathrm{~m}^{-1}\right) \cdot{ }^{17,46,47,68-71}$ We note that, although the results presented here correspond to graphene dispersions prepared from graphite foil, graphene dispersions could also be obtained by anodic exfoliation of other types of graphite in the presence of SNDS, specifically, from HOPG and natural graphite powder (see Fig S13, Table S1 and accompanying text in ESI $\dagger$ ).

\subsection{Multifunctional electrolytes for the preparation of oxide-free anodically exfoliated graphene:} implications and mechanism

In addition to identifying a number of chemical species that are effective as both electrolyte and dispersant in the anodic preparation of graphene in aqueous medium, the previous results have demonstrated that such a method is able to afford graphene nanosheets with structural and chemical characteristics comparable to those of graphene flakes produced by direct liquid-phase exfoliation 
of graphite (i.e., very low oxygen and defect contents). In our opinion, this finding is important for two reasons. First, it has the potential to broaden significantly the scope of application of anodically exfoliated graphenes, which has been deemed to be somewhat limited as a result of the substantial oxidation and relatively low structural quality usually associated to this graphene type. ${ }^{19,20}$ Thus, through modulation of its oxygen and defect content, anodic graphene could be targeted either for practical uses that are generally explored with high quality flakes, such as those obtained by direct liquid-phase or cathodic exfoliation approaches (e.g., in applications requiring high electrical or thermal conductivity), or alternatively, for uses where an extensive oxidation of the material, similar to that of graphene oxides, is desirable (e.g., to allow covalent functionalization with biomolecules towards theranostic applications). In addition, aqueous anodic exfoliation boasts significant advantages over other top-down, potentially scalable production methods. For example, it does not require the use of harsh chemicals (compared to the graphite oxide route) or organic solvents (as opposed to cathodic exfoliation), and affords higher yields and degrees of exfoliation (thinner flakes) relative to direct liquid-phase exfoliation.

Second, contrary to previous assumptions, ${ }^{19,20}$ the mentioned finding proves that significant oxidation of the graphene layers is neither a strictly required step nor an inevitable consequence of the aqueous anodic exfoliation method. As discussed above, anodic exfoliation is thought to be made possible by pre-expansion of the graphite edges to allow an efficient intercalation of anions from the electrolyte. In turn, such a pre-expansion is believed to be the result of attack of the graphite edges by hydroxyl and oxygen radicals generated through anodic oxidation of water molecules. $^{32,34}$ It would then be conceivable that the only stringent requirement for anodic intercalation and exfoliation to take place efficiently is that the edges of the graphene layers, but not their interiors (i.e., their basal planes), become oxidized, even though in practice basal plane oxidation occurs for the highly oxidized anodic graphenes that are usually reported in the literature. Because just a very small fraction of carbon atoms are expected to be located at the edge of graphene nanosheets with lateral sizes of a few hundred nanometers or larger, this implies that 
anodic exfoliation should be possible with minimal oxidation of the resulting graphenes, as actually demonstrated in the present work with, e.g., SNDS.

The main question to elucidate in connection with these results is why oxidation of the graphene flakes could be largely prevented with some electrolytes, especially SNDS. To this end, we note that intercalation of anions from the electrolyte within the graphene layers during the anodic process is probably accompanied by the co-intercalation of a number of water molecules surrounding each anion (hydrated anion). Oxidation of these water molecules at the high anodic potential applied (10 V) would generate the hydroxyl and oxygen radicals that in turn oxidize the graphene layers. However, in some cases oxidation of the anion itself would also be expected to take place. For instance, organic compounds and especially polycyclic aromatic hydrocarbons (PAHs), including sulfonated PAHs, are particularly prone to anodic oxidation in aqueous medium as a result of their relatively low oxidation potential. ${ }^{72-74}$ In fact, electrolytic oxidation methods have been successfully deployed for the degradation of organic compounds in wastewaters. ${ }^{75}$ On this ground, it is reasonable to assume that either (1) preferential anodic oxidation of the PAHs hinders the oxidation of water molecules and hence the generation of hydroxyl/oxygen radicals or, as a more likely possibility, (2) the PAHs do not avert water oxidation but act as scavengers of the resulting hydroxyl/oxygen radicals, leading to their own oxidation (to give, e.g., hydroxylated PAHs). As a result of either of these two processes, oxidation of the graphene layers would be prevented. Therefore, the use of readily oxidizable electrolytes (e.g., SNDS) can be an effective strategy to avoid the oxidation of anodically exfoliated graphenes and thus yield very high quality materials. The actual performance of any given oxidizable electrolyte towards this end is believed to depend on both thermodynamic and kinetic factors, but an analysis in this regard is beyond the scope of the present work. By contrast, non- or hardly oxidizable electrolytes (e.g., SS) will not be able to impede oxidation of the graphene layers, leading to the well-known products of relatively high oxygen content and low structural quality. The main processes thought to be involved in these two situations, as well the as the consequences for the resulting graphene nanosheets, are schematically 
depicted in Fig. 5. From these results it can be concluded that several of the investigated electrolytes, most significantly SNDS, boast not just a dual but a triple functionality in the anodic preparation of graphene: (1) exfoliating electrolyte, (2) colloidal dispersant and (3) sacrificial agent to prevent oxidation of the flakes.

\subsection{Synthesis and catalytic activity of anodically exfoliated graphene-Pt NP hybrids}

Hybrid nanostructures consisting of metallic and other types of NPs supported onto graphene are currently the focus of intense research efforts due to their strong potential for application in, e.g., catalysis or energy storage. ${ }^{76}$ However, the majority of such efforts involve the use of GO nanosheets, whereas other graphene types have received much less attention. This can be attributed to the fact that the oxygen functional groups present in GO at very high densities serve as efficient anchoring sites for the NPs. In the case of electrolytically exfoliated graphene, its combination with NPs to afford functional hybrids has just started to be explored, ${ }^{63}$ where polyaniline was employed as a linker to physically couple both components. We attempted the growth of Pt NPs on graphene flakes prepared by electrolytic exfoliation with the non-amphiphilic, commonly used electrolyte SS, which gives a significantly oxidized product $(\mathrm{O} / \mathrm{C}$ ratio 0.11 , see Table 1$)$. The procedure was based on reduction of a metal salt $\left(\mathrm{K}_{2} \mathrm{PtCl}_{4}\right)$ with $\mathrm{NaBH}_{4}$ in a water-isopropanol dispersion of exfoliated flakes. However, TEM imaging (see Fig. S14 of the ESI $\dagger$ ) indicated that very few particles were able to associate to the flakes suggesting a lack of efficient anchoring sites for anodically exfoliated graphenes even if there are relatively well oxidized in this case. Thus, for the anodically exfoliated graphenes with very low extent of oxidation prepared here using certain electrolytes/dispersants, the need for a linker to promote NP anchoring to the graphene support appears to be necessary. Rather than resorting to an additional component, we hypothesized that the very same chemical species used as electrolyte, dispersant and sacrificial agent (e.g., SPTS and SNDS) could also play the role of effective linker. These species must be strongly adsorbed on the graphene surface through $\pi-\pi$ interactions and their ionic (sulfonate) groups are expected to 
promote the nucleation and attachment of the NPs by acting as anchors for the precursor metal cationic species. To verify such a hypothesis, we synthesized Pt NPs by reduction of $\mathrm{K}_{2} \mathrm{PtCl}_{4}$ in an aqueous dispersion of either SPTS- or SNDS-stabilized graphene flakes (see Experimental section for details). As exemplified in Fig. 6a and c for SPTS- and SNDS-based samples, respectively, TEM imaging confirmed the decoration of the anodically exfoliated graphene flakes with large numbers of NPs. An analysis of their size (see histograms in Fig. $6 b$ and d) revealed that they were typically between 1 and $5 \mathrm{~nm}$ in diameter, with an average size of 1.9 \pm 0.8 (SPTS) and $3 \pm 1$ (SNDS) nm. No stand-alone NPs were observed in the TEM images, indicating that they were firmly anchored to the graphene support. XPS measurements demonstrated that the NPs were made up of Pt in metallic form. Fig. 6e shows the high resolution Pt 4 f core level spectrum for the SNDS-based sample (results for the SPTS-based hybrid were similar and are not shown). The spectrum consisted of two slightly asymmetrical peaks (doublet band) located at binding energies of 71.3 and $74.6 \mathrm{eV}$, which could be ascribed to the $4 \mathrm{f}_{7 / 2}$ and $4 \mathrm{f}_{5 / 2}$ levels of $\mathrm{Pt}^{0}$, respectively. ${ }^{77}$ No significant peaks associated to oxidized $\mathrm{Pt}$ (e.g., $\mathrm{Pt}^{4+}$ ) were noticed. The Pt/graphene mass ratio in the hybrids was determined by ICP-MS, yielding values of 4.0\% (SPTS) and 7.9\% (SNDS).

Among other potential applications, the anodically exfoliated graphene-Pt NP hybrids reported here could find use as catalytic systems. Their catalytic activity was evaluated on the basis of two model reactions carried out in aqueous medium at room temperature, namely the reduction of 4-NP and 4-NA with $\mathrm{NaBH}_{4}$ to yield 4-AP and $p$-PDA, respectively. Both reactions are thermodynamically favorable but their kinetics is restricted by significant activation barriers, so that they need to be catalytically promoted. In addition to their practical relevance in different areas, including the synthesis of pharmaceutical compounds or the management of recalcitrant pollutants, they are commonly studied benchmark reactions in the assessment of metal-based catalysts. ${ }^{78,79}$ The reaction progress can be readily monitored through UV-vis absorption spectroscopy in both cases. Fig. 7a shows the characteristic absorption spectrum of 4-NP at the slightly acidic pH of deionized water (black trace), which displays a strong band at $\sim 316 \mathrm{~nm}$. In the basic medium 
generated by the presence of the reductant $\mathrm{NaBH}_{4}, 4-\mathrm{NP}$ deprotonates to give the 4-nitrophenoxide anion, whose main absorption band is located at $400 \mathrm{~nm}$ (green trace). Reduction of 4nitrophenoxide with $\mathrm{NaBH}_{4}$ yields the 4-aminophenoxide anion, which exhibits absorption peaks at $\sim 295 \mathrm{~nm}$ and below but lacks absorbance at $400 \mathrm{~nm}$ (red trace). Therefore, reduction of 4-NP to 4AP can be kinetically monitored by the decrease in absorbance at $400 \mathrm{~nm}$. A similar reasoning leads to the selection of absorbance at $380 \mathrm{~nm}$ to follow the reduction of 4-NA (Fig. 7b, orange trace) to $p$-PDA (blue trace).

Figs. 7c and d show typical kinetic curves for the reduction of 4-NP and 4-NA, respectively, in the presence of the anodically exfoliated graphene-Pt NP hybrids. Reaction completion was generally seen to occur in less than $8 \mathrm{~min}$. No significant reduction took place in the absence of the hybrids or in the presence of only the graphene flakes, demonstrating the catalytic role of the metal component. Considering that the experimental kinetic profiles could be fitted to an exponential decay function (see Figs. 7c and d) and that $\mathrm{NaBH}_{4}$ was used to a large excess, so that its concentration could be regarded to remain constant throughout the reaction, the catalyzed reduction was best described by pseudo-first-order kinetics with respect to the substrate (4-NP or 4-NA), i.e., $\frac{\mathrm{d}[\mathrm{subs}]}{\mathrm{dt}}=-\mathrm{k}_{\mathrm{app}}[\mathrm{subs}]$

, where [subs] is the substrate concentration and $\mathrm{k}_{\mathrm{app}}$ is the apparent reaction rate constant. Typical values of $k_{a p p}$ measured for the present hybrids were in the range of $1-2 \times 10^{-2} \mathrm{~s}^{-1}(4-\mathrm{NP})$ and $2-$ $6 \times 10^{-2} \mathrm{~s}^{-1}$ (4-NA). To allow direct comparisons with other catalysts reported in the literature, the catalytic activity of the hybrids was quantified in terms of turnover frequency (TOF), defined as the number of substrate moles converted per mole of $\mathrm{Pt}$ used in the reaction per unit time. The calculated TOF values are given in Table 2, together with those of some of the best performing Pt NP-based catalysts documented beforehand towards $4-\mathrm{NP}^{46,80-83}$ and $4-\mathrm{NA}^{46,84-86}$ reduction. The catalytic activity of our graphene-Pt NP hybrids was similar (in the case of 4-NP reduction) or even higher (4-NA reduction) than that of the most efficient catalysts developed to date. These results 
highlight the strong potential of the electrolytically exfoliated graphenes developed here for use as catalyst supports.

\section{Conclusions}

We have identified a number of chemical species of amphiphilic nature that can be used as multifunctional electrolytes towards the electrolytic (anodic) exfoliation of graphite in water to give graphene flakes with much improved characteristics. First, a pool of amphiphiles (specifically, sulfonate- and sulfate-containing hydrocarbons) with the potential to exhibit a dual role as exfoliating (intercalating) electrolyte and colloidal dispersant in aqueous medium was evaluated. Benchmark tests designed to quantify the overall performance of the amphiphiles in fulfilling such a dual role indicated that successful species possess an appropriate balance between the number of their ionic groups and the size of their hydrophobic component. Second, in addition to acting as electrolyte and dispersant, several of the amphiphiles were also effective in preventing to a large extent the oxidation and structural degradation of the graphene flakes that is usually associated to anodic exfoliation. As a result, hardly oxidized graphenes of a similar quality to that of flakes produced via direct, ultrasound- or shear-induced exfoliation of graphite could be obtained by the anodic route for the first time. This highly relevant characteristic was rationalized on the basis of the ability of some amphiphiles to function as scavengers of the reactive oxygen species that are thought to attack and oxidize the graphene lattice during exfoliation, thus laying down a general strategy to overcome one of the main drawbacks of the anodic approach. Finally, the amphiphiles were also efficient linkers for the anchoring of metal nanoparticles onto the graphene flakes, yielding hybrids that exhibited a very high catalytic activity in the reduction of nitroarenes. We envisage this type of multifunctional electrolytes to be key enabling elements in the future practical implementation of high quality electrolytically exfoliated graphene.

\section{Acknowledgements}


Financial support from the Spanish Ministerio de Economía y Competitividad (MINECO) and the European Regional Development Fund (ERDF) through project MAT2011-26399 is gratefully acknowledged. Partial funding by Plan de Ciencia, Tecnología e Innovación (PCTI) 2013-2017 del Principado de Asturias and the ERDF (project GRUPIN14-056) is also acknowledged. J.M.M. and M.A.-V. are grateful to the Spanish Ministerio de Educación, Cultura y Deporte (MECD) and MINECO, respectively, for their pre-doctoral contracts.

\section{References}

1 A. K. Geim, K. S. Novoselov, Nat. Mater., 2007, 6, 183-191.

2 A. K. Geim, Science, 2009, 324, 1530-1534.

3 K. S. Novoselov, V. I. Fal'ko, L. Colombo, P. R. Gellert, M. G. Schwab, K. Kim, Nature, 2012, 490, 192-200.

4 A. C. Ferrari, F. Bonaccorso, V. I. Fal'ko, K. S. Novoselov, S. Roche, P. Bøggild, S. Borini, F. Koppens, V. Palermo, N. Pugno J. A. Garrido, R. Sordan, A. Bianco, L. Ballerini, M. Prato, E. Lidorikis, J. Kivioja, C. Marinelli, T. Ryhänen, A. Morpurgo, J. N. Coleman, V. Nicolosi, L. Colombo, A. Fert, M. Garcia-Hernandez, A. Bachtold, G. F. Schneider, F. Guinea, C. Dekker, M. Barbone, C. Galiotis, A. Grigorenko, G. Konstantatos, A. Kis, M. Katsnelson, C. W. J. Beenakker, L. Vandersypen, A. Loiseau, V. Morandi, D. Neumaier, E. Treossi, V Pellegrini,M. Polini, A. Tredicucci, G. M. Williams, B. H. Hong, J. H. Ahn, J. M. Kim, H. Zirath, B. J. van Wees, H. van der Zant, L. Occhipinti, A. Di Matteo, I. A. Kinloch, T. Seyller, E. Quesnel, X. Feng, K. Teo, N. Rupesinghe, P. Hakonen, S. R. T. Neil, Q.Tannock, T. Löfwander, J. Kinaret, Nanoscale, 2015, 7, 4598-4810.

5 M. Cai, D. Thorpe, D. H. Adamson, H. C. Schniepp, J. Mater. Chem., 2012, 22, 24992-25002.

6 F. Bonaccorso, A. Lombardo, T. Hasan, Z. Sun, L. Colombo, A.C. Ferrari, Mater. Today, 2012, 15, 564-589.

7 Y. L. Zhong, Z. Tian, G. P. Simon, D. Li, Mater. Today, 2015, 18, 73-78.

8 S. Park, R. S. Ruoff, Nat. Nanotechnol., 2009, 4, 217-224.

9 K. P. Loh, Q. Bao, G. Eda, M. Chhowalla, Nat. Chem., 2010, 2, 1015-1024.

10 L. Feng, L. Wu, X. Qu, Adv. Mater., 2013, 25, 168-186.

11 V. Chabot, D. Higgins, A. Yu, X. Xiao, Z. Chen, J. Zhang, Energy Environ. Sci., 2014, 7, 1564 1596. 
12 A. Esfandiar, A. Irajizad, O. Akhavan, S. Ghasemi, M. R. Gholami, Int. J. Hydrogen Energy, 2014, 39, 8169-8179.

13 C. Mattevi, G. Eda, S. Agnoli, S. Miller, K.A. Mkhoyan, O. Celik, D. Mastrogiovanni, G. Granozzi, E. Garfunkel, M. Chhowalla, Adv. Funct. Mater., 2009,19, 2577-2583.

14 R. Rozada, J. I. Paredes, M. J. López, S. Villar-Rodil, I. Cabria, J. A. Alonso, A. MartínezAlonso, J. M. D. Tascón, Nanoscale, 2015, 7, 2374-2390.

15 J. N. Coleman, Acc. Chem. Res., 2013, 46, 14-22.

16 A. Ciesielski, P. Samorì, Chem. Soc. Rev., 2014, 43, 381-398.

17 K. R. Paton, E. Varrla, C. Backes, R. J. Smith, U: Khan, A. O’Neill, C. Boland, M. Lotya, O. M. Istrate, P. King, T. Higgins, S. Barwich, P. May, P. Puczkarski, I. Ahmed, M. Moebius, H. Pettersson, E. Long, J. Coelho, S. E. O’Brien, E. K. McGuire, B. Mendoza Sanchez, G. S. Duesberg, N. McEvoy, T. J. Pennycook, C. Downing, A. Crossley, V. Nicolosi1, J. N. Coleman, Nat. Mater., 2014, 13, 624-630.

18 C. T. J. Low, F. C. Walsh, M. H. Chakrabarti, M. A. Hashim, M. A. Hussain, Carbon, 2013, 54, $1-21$.

19 H. J. Salavagione, J. Mater. Chem. A, 2014, 2, 7138-7146.

20 A. M. Abdelkader, A. J. Cooper, R. A. W. Dryfe, I. A. Kinloch, Nanoscale, 2015, 7, 6944-6956.

21 J. Liu, M. Notarianni, G. Will, V.T. Tiong, H. Wang, N. Motta, Langmuir, 2013, 29, 1330713314.

22 Z.-S. Wu, Z. Liu, K. Parvez, X. Feng, K. Müllen, Adv. Mater., 2015, 27, 3669-3675.

23 L. Zhang, H. Huang, H. Yin, Y. Xia, J. Luo, C. Liang, Y. Gan, X. Tao, W. Zhang, J. Mater. Chem. A, 2015, 3, 16513-16519.

24 J. Liu, C. K. Poh, D. Zhan, L. Lai, S. H. Lim, L. Wang, X. Liu, N. G. Sahoo, C. Li, Z. Shen, J. Lin, Nano Energy, 2013, 2, 377-386.

25 K. S. Rao, J. Sentilnathan, H.-W. Cho, J.-J. Wu, M. Yoshimura, Adv. Funct. Mater., 2015, 25, 298-305.

26 P. Tripathi, Ch. R. P. Patel, A. Dixit, A. P. Singh, P. Kumar, M. A. Shaz, R. Srivastava, G. Gupta, S. K. Dhawan, B. K. Gupta, O. N. Srivastava, RSC Adv., 2015, 5, 19074-19081.

27 J. Wang, K. K. Manga, Q. Bao, K. P. Loh, J. Am. Chem. Soc., 2011, 133, 8888-8891.

28 M. Zhou, J. Tang, Q. Cheng, G. Xu, P. Cui, L.-C. Qin, Chem. Phys. Lett., 2013, 572, 61-65.

29 A. M. Abdelkader, I. A. Kinloch, R. A. W. Dryfe, ACS Appl. Mater. Interfaces, 2014, 6, 16321639.

30 A.T. Najafabadi, E. Gyenge, Carbon, 2015, 84, 449-459.

31 M. Zhao, X.-Y. Guo, O. Ambacher, C. E. Nebel, R. Hoffmann, Carbon, 2015, 83, 128-135. 
32 J. Lu, J. Yang, J. Wang, A. Lim, S. Wang, K. P. Loh, ACS Nano, 2009, 3, 2367-2375.

33 C.-Y. Su, A.-Y. Lu, Y. Xu, F.-R. Chen, A. N. Khlobystov, L.-J. Li, ACS Nano, 2011, 5, 2332 2339.

34 K. Parvez, Z.-S. Wu, R. Li, X. Liu, R. Graf, X. Feng, K. Müllen, J. Am. Chem. Soc., 2014, 136, 6083-6091.

35 A. T. Najafabadi, E. Gyenge, Carbon, 2014, 71, 58-69.

36 K. Chen, D. Xue, J. Colloid Interface Sci.,2014, 436, 41-46.

37 Y. Hernandez, M. Lotya, D. Rickard, S. D. Bergin, J. N. Coleman, Langmuir, 2010 26, 32083213.

38 S. Park, J. An, I. Jung, R. D. Piner, S. J. An, X. Li, A. Velamakanni, R. S. Ruoff, Nano Lett., 2009, 9, 1593-1597.

39 S. Villar-Rodil, J. I. Paredes, A. Martínez-Alonso, J. M. D. Tascón, J. Mater. Chem.,2009, 19, 3591-3593.

40 M. Alanyalıŏlu, J. J. Segura, J. Oró-Solè, N. Casañ-Pastor, Carbon, 2012, 50, 142-152.

41 J. P. Mensing, T. Kerdcharoen, C. Sriprachuabwong, A. Wisitsoraat, D. Phokharatkul, T. Lomas, A. Tuantranont, J. Mater. Chem , 2012, 22, 17094-17099.

42 T. Kuila, P. Khanra, N. H. Kim, S. K. Choi, H. J. Yun, J. H. Lee, Nanotechnology, 2013, 24, 365706.

43 C.-H. Chen, S.-W. Yang, M.-C. Chuang, W.-Y. Woon, C.-Y. Su, Nanoscale, 2015, 7, 1536215373.

44 J. M. Munuera, J. I. Paredes, S. Villar-Rodil, M. Ayán-Varela, A. Pagán, S. D. AznarCervantes, J. L. Cenis, A. Martínez-Alonso, J. M. D. Tascón, Carbon, 2015, 94, 729-739.

45 Z. Sun, J. Masa, Z. Liu, W. Shuhmann, M. Muhler, Chem. Eur. J., 2012, 18, 6972-6978.

46 M. Ayán-Varela, J. I. Paredes, L. Guardia, S. Villar-Rodil, J. M. Munuera, M. Díaz-González, C. Fernández-Sánchez, A. Martínez-Alonso, J. M. D. Tascón, ACS Appl. Mater. Interfaces, 2015, 7, 10293-10307.

47 L. Guardia, M. J. Fernández-Merino, J. I. Paredes, P. Solís-Fernández, S. Villar-Rodil, A. Martínez-Alonso, J. M. D. Tascón, Carbon, 2011, 49, 1653-1662.

48 M. J. Fernández-Merino, J. I. Paredes, S. Villar-Rodil, L. Guardia, P. Solís-Fernández, D. Salinas-Torres, D. Cazorla-Amorós, E. Morallón, A. Martínez-Alonso, J. M. D. Tascón, Carbon, 2012, 50, 3184-3194.

49 X. Lu, C. Zhao, Phys. Chem. Chem. Phys., 2013, 15, 20005-20009.

50 D. Parviz, S. Das, H. S. T. Ahmed, F. Irin, S. Bhattacharia, M. J. Green, ACS Nano, 2012, 6, 8857-8867. 
51 A. Schlierf, H. Yang, E. Gebremedhn, E. Treossi, L. Ortolani, L. Chen, A. Minoia, V. Morandi, P. Samorì, C. Casiraghi, D. Beljonne, V. Palermo, Nanoscale, 2013, 5, 4205-4216.

52 D. Li, M. B. Müller, S. Gilje, R .B. Kaner, G. G. Wallace, Nat. Nanotech., 2008, 3, 101-105.

53 J.-W. T. Seo, A. A. Green, A. L. Antaris, M. C. Hersam, J. Phys. Chem. Lett., 2011, 2, 10041008.

54 D. A. Skoog, F. J. Holler, T. A. Nieman, Principles of Instrumental Analysis, Hartcourt Brace \& Company, Philadelphia, 1998, ch. 13.

55 M. Lotya, Y. Hernandez, P. J. King, R. J. Smith, V. Nicolosi, L. S. Karlsson, F. M. Blighe, S. De, Z. Wang, I. T. McGovern, G. S. Duesberg, J. N. Coleman, J. Am. Chem. Soc., 2009, 131, 36113620.

56 H.-J. Butt, K. Graf, M. Kappl, Physics and Chemistry of Interfaces, Wiley-VCH, Weinheim, 2003.

57 M. S. Dresselhaus, G. Dresselhaus, Adv. Phys., 1981, 30, 139-326.

58 M. Noel, R. Santhanam, J. Power Sources, 1998, 72, 53-65.

59 W. Yan, M. M. Lerner, Carbon, 2004, 42, 2981-2987.

60 M. Lotya, P. J. King, U. Khan, S. De, J. N Coleman. ACS Nano, 2010, 4, 3155-3162.

61 Z. Y. Xia, S. Pezzini, E. Treossi, G. Giambastiani, F. Corticelli, V. Morandi, A. Zanelli, V. Bellani, V. Palermo, Adv. Funct. Mater., 2013, 23, 4684-4693.

62 L. Wu, W. Li, P. Li, S. Liao, S. Qiu, M. Chen, Y. Guo, Q. Li, C. Zhu, L. Liu, Small, 2014, 10, 1421-1429.

63 W. Wei, G. Wang, S. Yang, X. Feng, K. Müllen, J. Am. Chem. Soc., 2015, 137, 5576-5581.

64 A. J. Cooper, N. R. Wilson, I. A. Kinloch, R. A. W. Dryfe, Carbon, 2014, 66, 340-350.

65 U. Halim, C. R. Zheng, Y. Chen, Z. Lin, S. Jiang, R. Cheng, Y. Huang, X. Duan, Nat. Commun., 2013, 4, 2213.

66 M. A. Pimenta, G. Dresselhaus, M. S. Dresselhaus, L. G. Cançado, A. Jorio, R. Saito, Phys. Chem. Chem. Phys., 2007, 9, 1276-1291.

67 A. C. Ferrari, D. M. Basko, Nat. Nanotechnol., 2013, 8, 235-246.

68 S. De, P. J. King, M. Lotya, A. O’Neill, E. M. Doherty, Y. Hernandez, G. S. Duesberg, J. N. Coleman, Small, 2010, 6, 458-464.

69 U. Khan, A. O`Neill, M. Lotya, S. De, J. N. Coleman, Small, 2010, 6, 864-871.

70 V. Chabot, B. Kim, B. Sloper, C. Tzoganakis, A. Yu, Sci. Rep., 2013, 3, 1378.

71 L. Zhang, Z. Zhang, C. He, L. Dai, J. Liu, L. Wang, ACS Nano, 2014, 8, 6663-6670.

72 C.A. Martínez-Huitle, S. Ferro, Chem. Soc. Rev., 2006, 35, 1324-1340.

73 M. Panizza, M. Zolezzi, C. Nicolella, J. Chem. Technol. Biotechnol., 2006, 81, 225-232. 
74 J. Muff, E.G. Søgaard, Water Sci. Technol., 2010, 61, 2043-2051.

75 C.A. Martínez-Huitle, E. Brillas, Appl. Catal. B: Environ., 2009, 87, 105-145.

76 M. Khan, M. N. Tahir, S. F. Adil, H. U. Khan, M. R. H. Siddiqui, A. A. Al-warthan, W. Tremel, J. Mater. Chem. A, 2015, 3, 18753-18808.

77 B. V. Crist, Handbook of Monochromatic XPS Spectra, XPS International LLC, Mountain View, 2004, Vol. 1. The Elements and Native Oxides.

78 T. Aditya, A. Pal, T. Pal, Chem. Commun., 2015, 51, 9410-9431.

79 H. Hu, J.H. Xin, H. Hu, X. Wang, D. Miao, Y. Liu, J. Mater. Chem. A, 2015, 3, 11157-11182.

80 C. Kim, H. Lee, Catal. Commun., 2009, 10, 1305-1309.

81 Y. Dai, Y. Chai, Y. Sun, W. Fu, X. Wang, Q. Gu, T. H. Zeng, Y. Sun, J. Mater. Chem. A, 2015, 3, 125-130.

82 E. Kan, L. Kuai, W. Wang, B. Geng, Chem. Eur. J., 2015, 21, 13291-13296.

83 B. K. Barman, K. K. Nanda, Appl. Catal. A: Gen., 2015, 491, 45-51.

84 S. K. Ghosh, M. Mandal, S. Kundu, S. Nath, T. Pal, Appl. Catal. A: Gen., 2004, 268, 61-66.

85 M. M. Raju, D. K. Pattanayak, RSC Adv., 2015, 5, 59541-59549.

86 X. Zhang, D. Liu, L. Yang, L. Zhou, T. You, J. Mater. Chem. A, 2015, 3, 10031-10037. 
Table 1. Characteristics of anodically exfoliated graphenes obtained with several intercalating electrolytes. The apparent thickness of the graphene flakes was gathered by AFM. The $\mathrm{O} / \mathrm{C}$ atomic ratio was obtained from XPS survey spectra. $\mathrm{I}_{\mathrm{D}} / \mathrm{I}_{\mathrm{G}}$ values were obtained from the Raman spectra. The name, chemical structure and acronym and the different concentrations assayed for each species tested here as potential electrolyte/dispersant electrolyte are indicated. The concentration of graphene material in the final dispersion measured by UV-vis absorption spectroscopy is also given.

Electrolyte

Structure

[electrolyte [graphene]

Apparent $\mathrm{O} / \mathrm{C} \quad \mathrm{I}_{\mathrm{D}} / \mathrm{I}_{\mathrm{G}}$

] thickness

(acronym)

(M) $\quad\left(\mathrm{mg} \mathrm{mL}^{-1}\right) \quad(\mathrm{nm})$

Sodium sultate

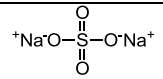

0.10

0.00

$2.1 \quad 0.11 \quad 1.0$

(SS)

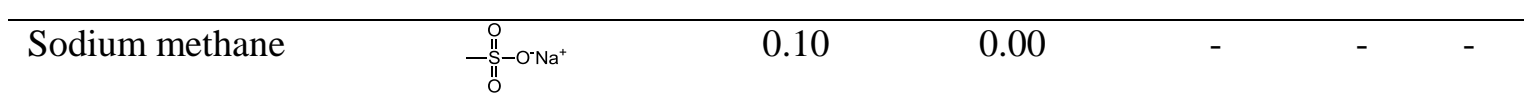
sulfonate

(SMS)

\begin{tabular}{|c|c|c|c|c|c|}
\hline Sodium bencene & 0.01 & 0.02 & 2.0 & 0.07 & 0.8 \\
\hline sulfonate & 0.05 & 0.19 & & 0.08 & 1.1 \\
\hline \multirow[t]{2}{*}{ (SBS) } & 0.10 & 0.40 & & 0.07 & 1.1 \\
\hline & 0.20 & 0.58 & & 0.09 & 0.8 \\
\hline Sodium bencene & 0.01 & 0.16 & 1.6 & 0.10 & 0.9 \\
\hline 1,3-disulfonate & 0.05 & 0.20 & & 0.11 & 1.2 \\
\hline \multirow[t]{2}{*}{ (SBDS) } & 0.10 & 0.42 & & 0.12 & 1.0 \\
\hline & 0.20 & 0.15 & & 0.10 & 0.9 \\
\hline Sodium $p$-toluene & 0.01 & 0.04 & 2.4 & 0.07 & 0.4 \\
\hline sulfonate & 0.05 & 0.13 & & 0.08 & 0.7 \\
\hline
\end{tabular}


(STS)

$\begin{array}{llll}0.10 & 0.14 & 0.08 & 0.6 \\ 0.20 & 0.51 & 0.07 & 0.8 \\ 0.50 & 0.55 & 0.07 & 0.8\end{array}$

Sodium $p$-toluate

0.10

0.00

(SPT)

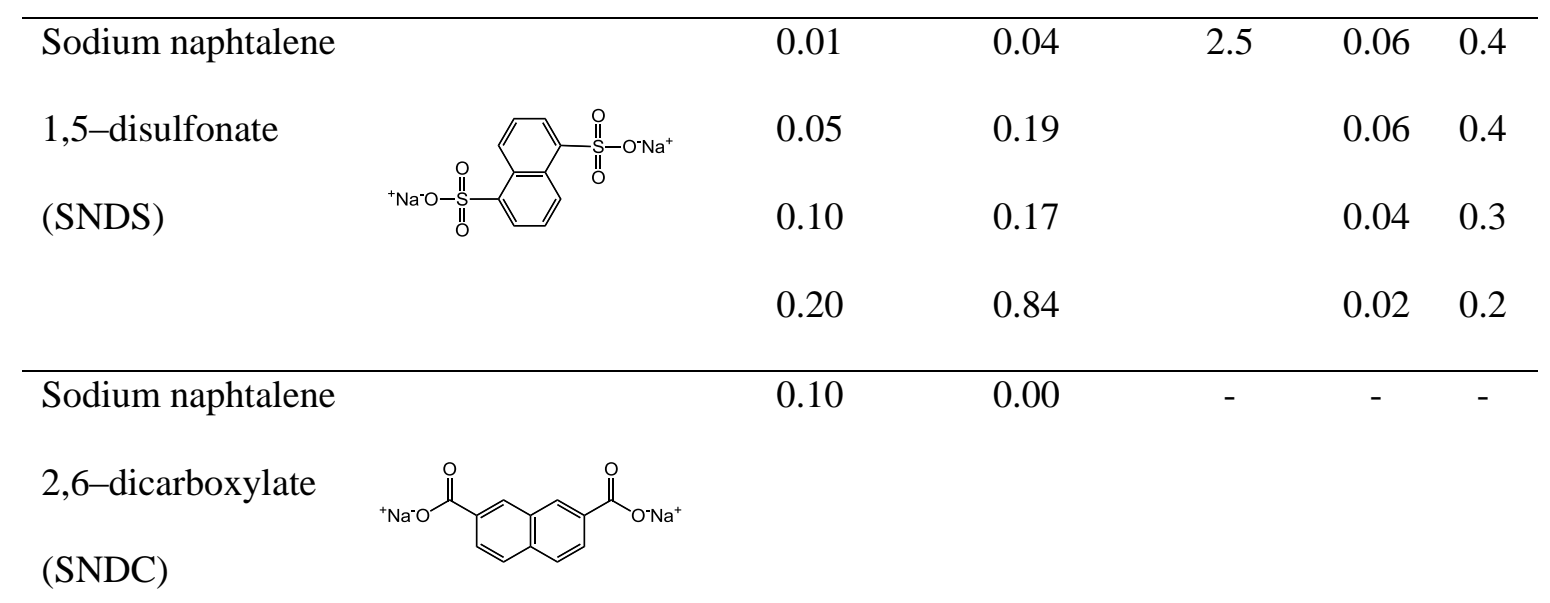

\begin{tabular}{|c|c|c|c|c|c|}
\hline Sodium naphtalene & 0.01 & 0.06 & 1.9 & 0.05 & 0.3 \\
\hline $1,3,(6,7)-$ & 0.05 & 0.37 & & 0.06 & 0.4 \\
\hline trisulfonate & 0.10 & 0.32 & & 0.05 & 0.3 \\
\hline (SNTS) & 0.20 & 0.40 & & 0.05 & 0.3 \\
\hline
\end{tabular}

Sodium pyrene

1-sulfonate

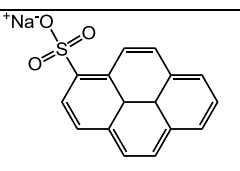

0.01

0.00

(SPS)

\begin{tabular}{|c|c|c|c|c|c|c|}
\hline Sodium pyrene & ${ }^{+} \mathrm{Na}^{-1} \mathrm{C}$ & 0.005 & 0.12 & 1.8 & 0.08 & 0.6 \\
\hline $1,3,6,8-$ & & 0.01 & 0.33 & & 0.06 & 0.6 \\
\hline tetrasulfonate & & 0.05 & 0.14 & & 0.06 & 0.3 \\
\hline (SPTS) & & & & & & \\
\hline Sodium dodecyl & & 0.05 & 0.04 & 2.1 & 0.10 & 1.1 \\
\hline
\end{tabular}




\begin{tabular}{lllllll} 
sulfate & 0.10 & 0.18 & 0.09 & 0.9 \\
Sodium dodecyl & 0.20 & 0.71 & 0.08 & 1.0 \\
bencene sulfate & 0.05 & 0.16 & 0.06 & 0.7 \\
(SDBS) & 0.10 & 0.42 & 0.10 & 0.9 \\
& 0.20 & 0.29 & 0.09 & 1.0 \\
& & 0.07 & 0.07 & 1.1 \\
\hline
\end{tabular}


Table 2. Comparison of turnover frequency (TOF) values for state-of-the-art Pt-based catalysts used for the reduction of 4-NP and 4-NA with $\mathrm{NaBH}_{4}$ in aqueous medium

Catalyst $\quad$ TOF $\left(\min ^{-1}\right) \quad$ Reference

Reduction of 4-NP

Anodically exfoliated graphene (SPTS)-Pt NP hybrid

$11 \quad$ Present work

Anodically exfoliated graphene (SNDS)-Pt NP hybrid

$14 \quad$ Present work

Pt NPs supported on ultrasound-exfoliated graphene

11

46

Surfactant-capped Pt nanocubes

2.5

80

Pt NPs supported on $\mathrm{Fe}_{2} \mathrm{O}_{3} / \mathrm{N}$-doped RGO hybrid

10.8

81

Pt NPs supported on $\mathrm{Al}_{2} \mathrm{O}_{3}$ microspheres

0.6

82

RGO-supported Pt NPs

10.4

83

Reduction of 4-NA

Anodically exfoliated graphene (SPTS)-Pt NP hybrid

$57 \quad$ Present work

Anodically exfoliated graphene (SNDS)-Pt NP hybrid

23

Present work

Pt NPs supported on ultrasound-exfoliated graphene

23

46

Bimetallic Pt-Ni NPs

$8.7^{\mathrm{a}}$

84

Pt NPs supported on RGO

14.5

85

Pt NPs embedded in RGO aerogel

0.8

86

\footnotetext{
${ }^{\mathrm{a}}$ TOF value calculated considering only the molar amount of Pt present in the bimetallic NPs
} 

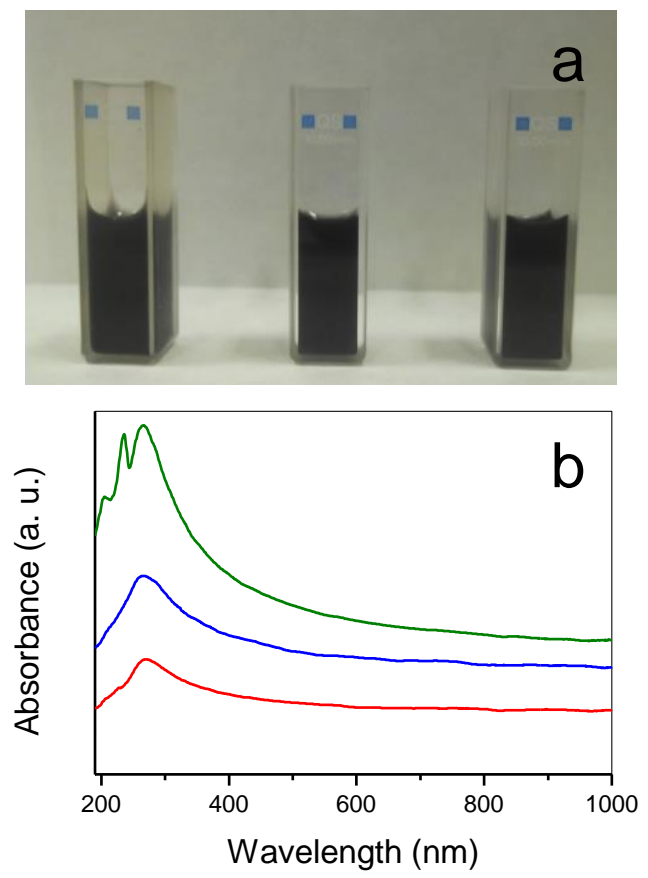

Figure 1. (a) Digital photograph of graphene suspensions obtained in the presence of different electrolytes (from left to right): SNTS, SPTS, and SNDS. (b) UV-vis absorption spectra of the graphene suspensions in (a): SNTS (green trace), SPTS (blue trace), and SNDS (red trace). 

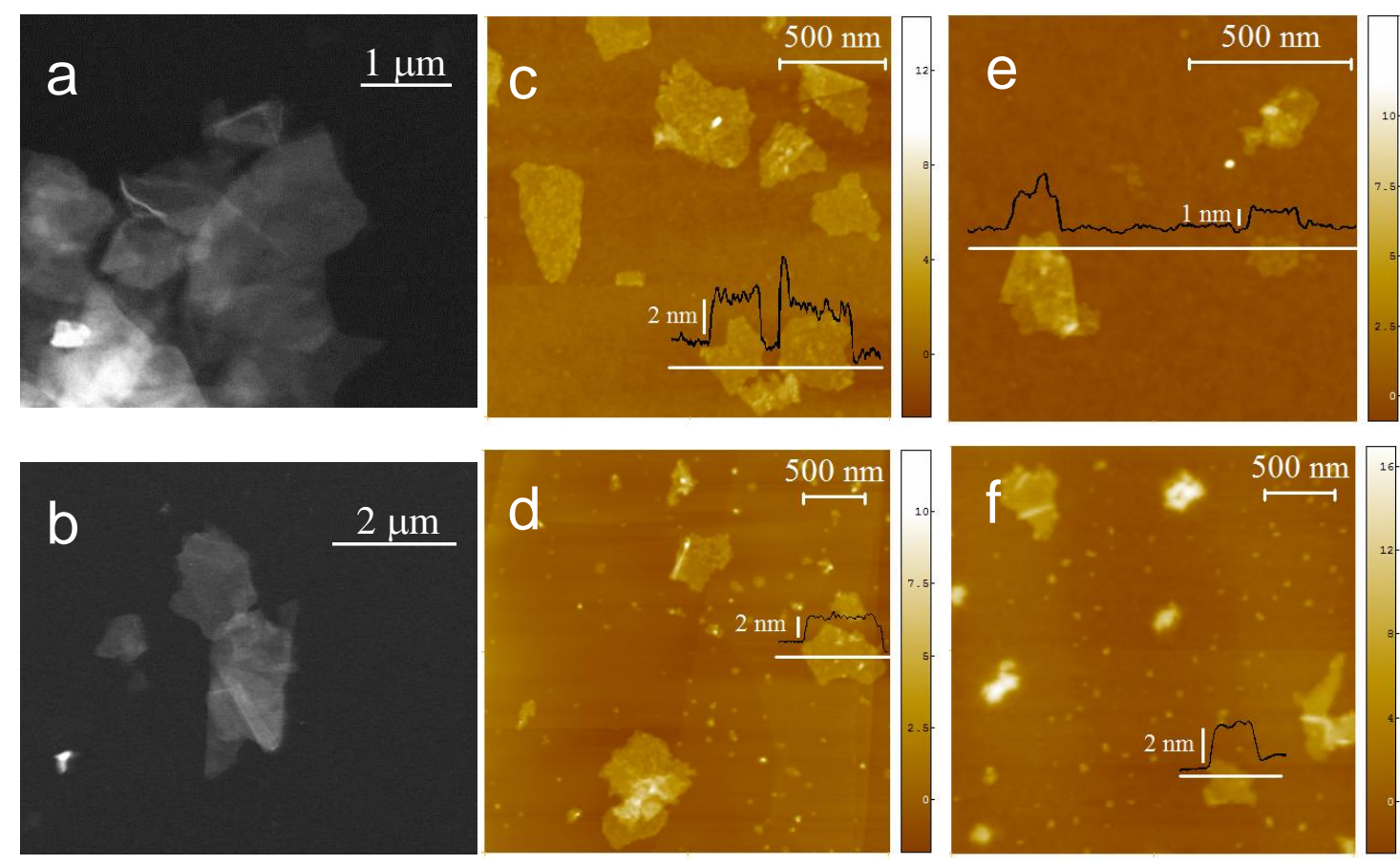

Figure 2. Representative STEM images of the graphene dispersions obtained with 0.2 M SNDS (a) and $0.01 \mathrm{M}$ SPTS (b). Representative AFM images of graphene dispersions prepared with 0.1 M SS (c, dispersion prepared in water-isopropanol mixture and shown for comparison purposes), 0.01 M SPTS (d), 0.05 M SBDS (e) and 0.2 M SNDS (f). A typical line profile taken along the marked white line is shown superimposed on each image. 


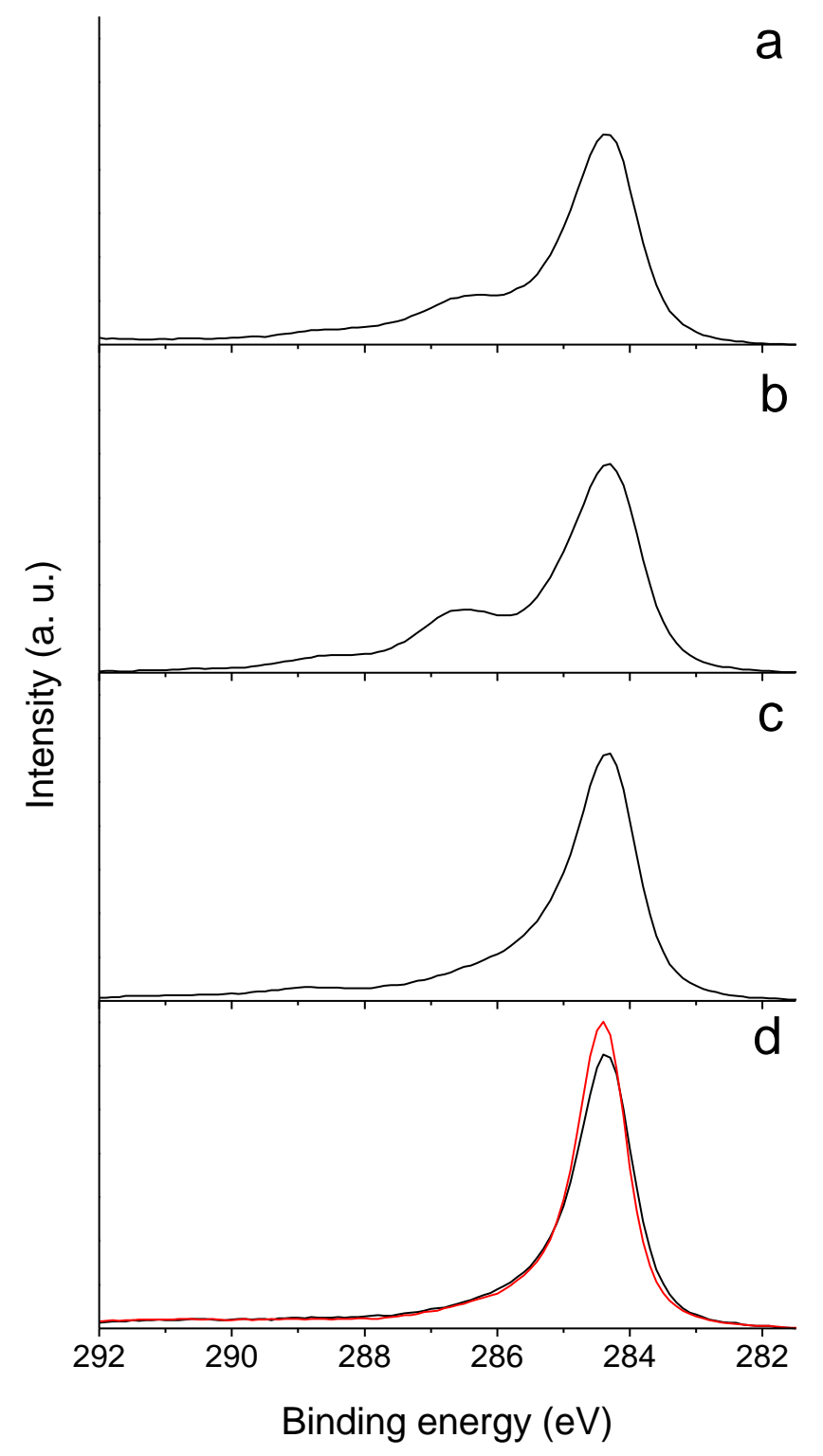

Figure 3. High resolution C 1s core level XPS spectra for graphene obtained with SS (a), SBDS (b), SPTS (c), and SNDS (d). For the sake of comparison, the C 1s spectrum of a sample obtained by direct exfoliation of graphite powder through sonication in a sodium cholate aqueous solution is also shown (red trace). 


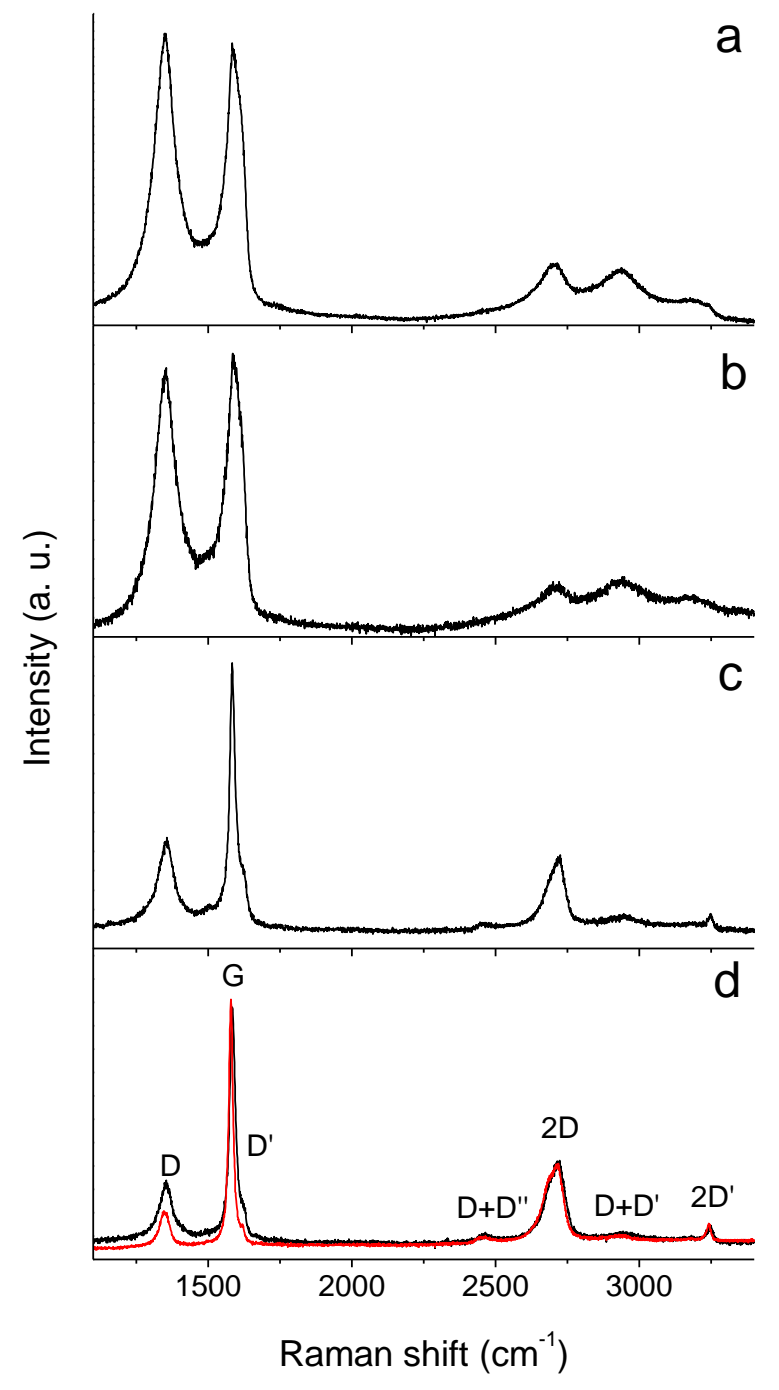

Figure 4. Raman spectra for graphene samples with SS (a), SBDS (b), SPTS (c), and SNDS (d). For the sake of comparison, the spectrum of a sample obtained by direct exfoliation of graphite powder through sonication in a $0.1 \mathrm{mg} \mathrm{mL}^{-1}$ sodium cholate aqueous solution is also shown (red trace). The main peaks are labeled for clarity. 


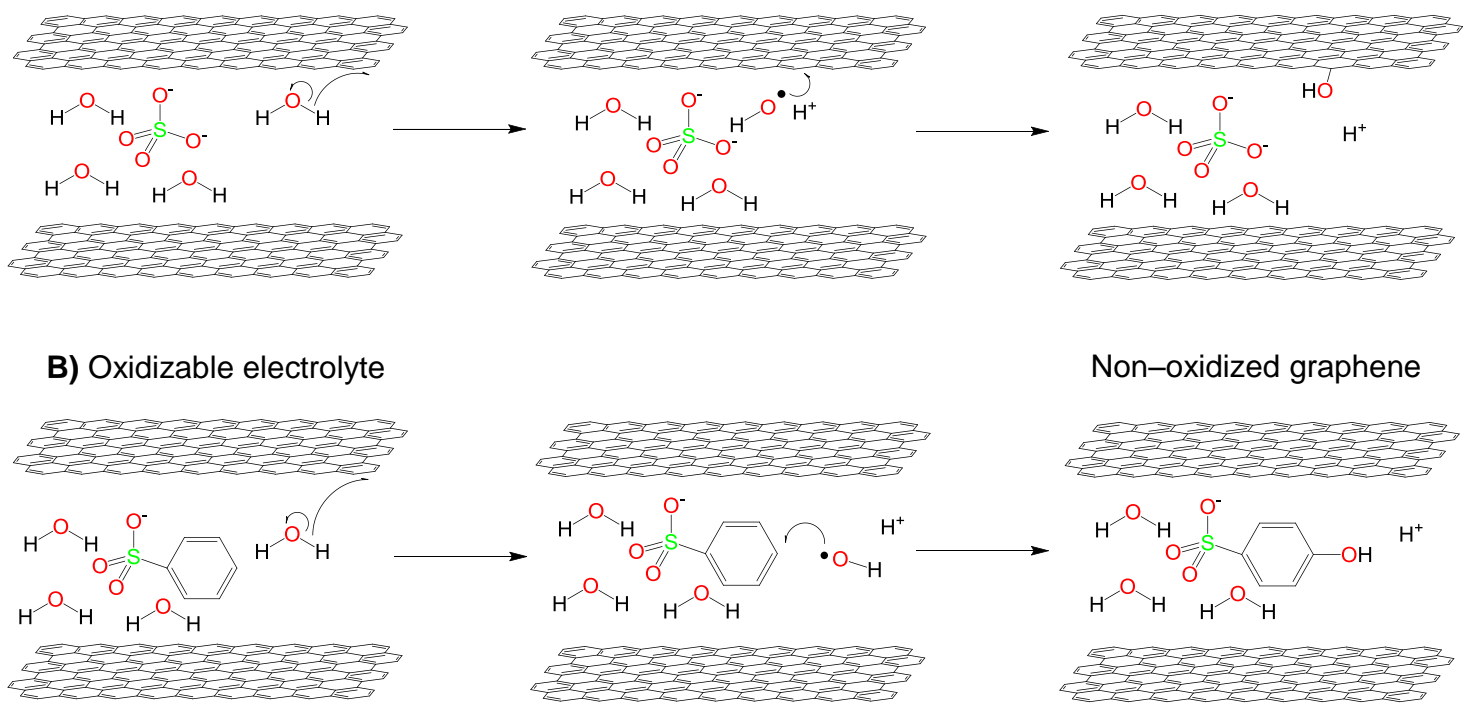

Figure 5. A) When graphite is anodically exfoliated in water in the presence of a nonoxidizable electrolyte such as sodium sulphate (SS), water molecules oxidize at the graphite anode, giving rise to highly reactive hydroxyl and oxygen radicals that then attack the graphene layers. As a result, the SS-derived graphene flakes are significantly oxidized. B) In the presence of a readily oxidizable electrolyte (an electrolyte with low oxidation potential), such as sodium benzenesulfonate (SBS), preferential anodic oxidation of electrolyte (to give, e.g., hydroxylated electrolyte) hinders the oxidation of graphene. Thus, the electrolyte acts as sacrificial agent preventing graphene oxidation. 

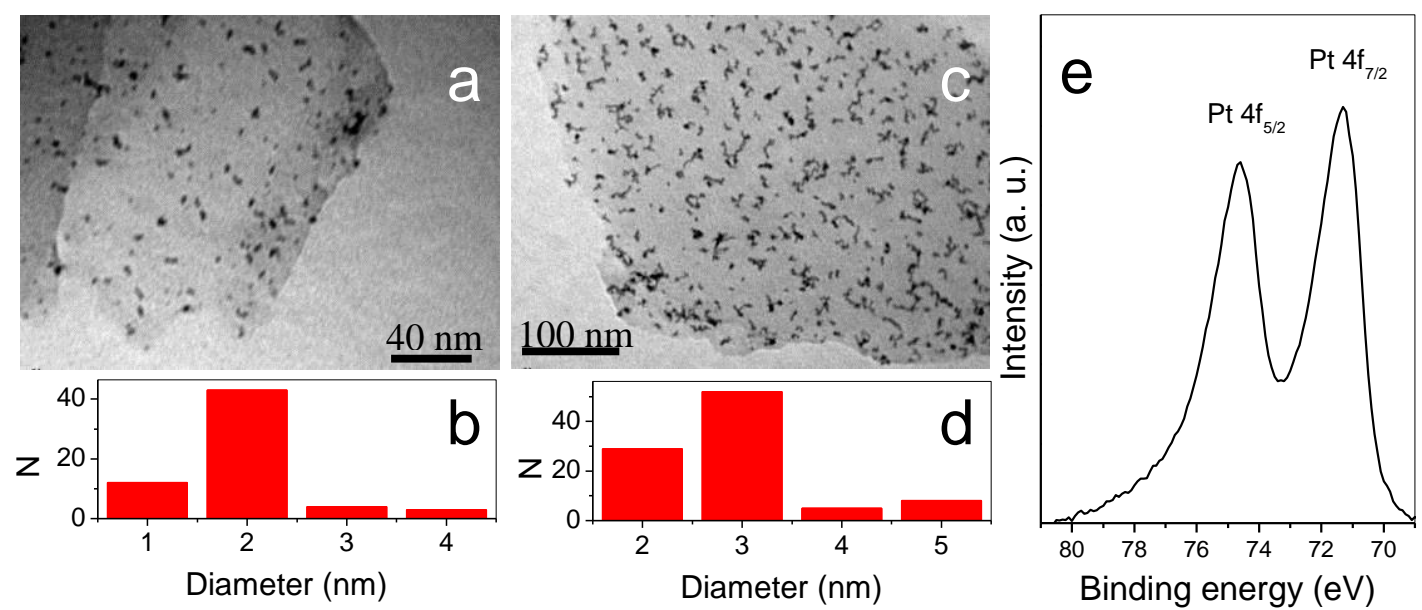

Figure 6. Representative TEM images of anodically exfoliated graphene-Pt NP hybrids obtained with SPTS (a) and SNDS (c). Histograms of nanoparticle size distribution derived from the TEM images for the graphene-Pt NP hybrids obtained with SPTS (b) and SNDS (d). (e) Representative high resolution XPS Pt 4f core level spectrum of the anodically exfoliated graphene-Pt NP hybrids. 

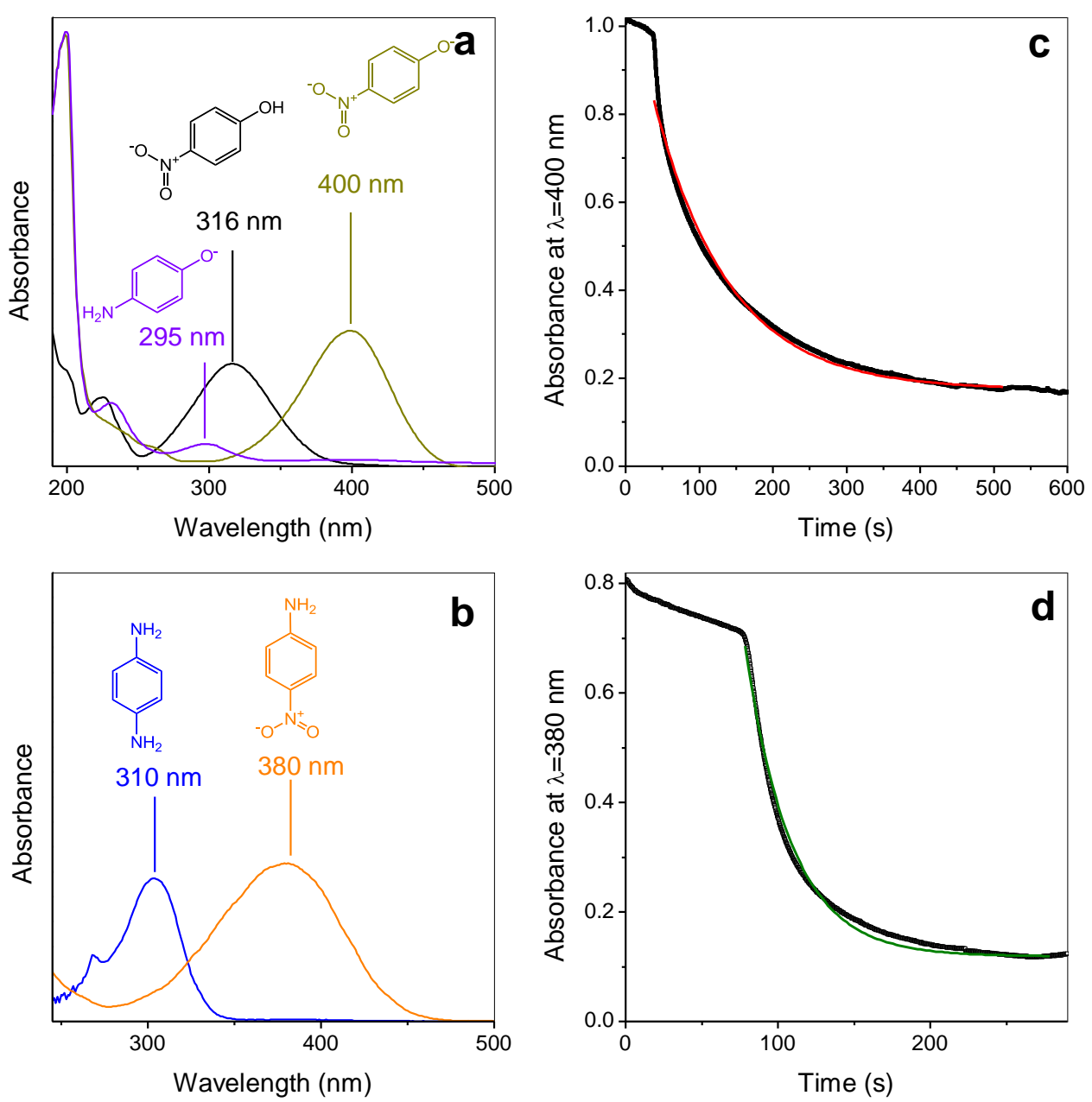

Figure 7. (a) UV-vis absorption spectra of 4-NP (black curve), 4-nitrophenoxide ion (dark yellow), and 4-aminophenoxide ion (violet). The absorption peak at $400 \mathrm{~nm}$ of 4nitrophenoxide is used to monitor its conversion to 4-aminophenoxide by reduction with $\mathrm{NaBH}_{4}$. (b) UV-vis absorption spectra of 4-NA (orange trace) and $p$-PDA (blue). The absorption peak at $380 \mathrm{~nm}$ of 4-NA is used to monitor the reaction progress. (c) Plot of absorbance at $400 \mathrm{~nm}$ vs. time for the reduction of 4-nitrophenoxide with $\mathrm{NaBH}_{4}$ in the presence of the anodically exfoliated graphene-Pt NP hybrid obtained with $0.01 \mathrm{M}$ SPTS. The experimental kinetic profile could be fitted to an exponential decay function, which is shown as an overlaid red line. (d) Plot of absorbance at $380 \mathrm{~nm}$ for the reduction of 4-NA with $\mathrm{NaBH}_{4}$ as catalyzed by the anodically exfoliated graphene-Pt 
NP hybrid obtained with 0.2 M SNDS. The experimental kinetic profiles could be fitted to a straight line which is shown as an overlaid green line. 